18 February 2011

\title{
Equally-Distributed Equivalent Utility, Ex Post Egalitarianism and Utilitarianism
}

\begin{abstract}
We provide an axiomatization of expected equally-distributed equivalent-utility social welfare functions in the context of Harsanyi's impartial observer theorem. For this family of social welfare functions, we show what additional axiom is necessary and sufficient for the observer to exhibit aversion to ex post inequality. We also relate this axiomatization to our axiomatization in a companion paper of generalized utilitarian social welfare functions. Given certain richness assumptions, the only social welfare functions that belong to both families are the utilitarian.

Keywords: utilitarianism, Harsanyi's (impartial) observer, social welfare function, ex post egalitarianism.
\end{abstract}

JEL Classification: D63, D71

Simon Grant

Department of Economics

Rice University

and

School of Economics

University of Queensland

Atsushi Kajii

Insitute of Economic Research

Kyoto University

Ben Polak

Department of Economics \& School of Management

Yale University

Zvi Safra

University of Exeter, The College of Management \& Tel Aviv University 


\section{Introduction}

In a companion paper (Grant et al, [6]), we revisited the setting of Harsanyi's [10, 11] utilitarian impartial observer theorem in which a society of individuals $\mathcal{I}$ has to choose among different social policies, each of which induces a probability distribution or 'lottery' $\ell$ over a set of social states $\mathcal{X}$. Each individual $i$ has preferences $\succsim_{i}$ over these lotteries. These preferences are known, and they differ.

To help choose among social policies, Harsanyi [10] proposed that each individual should imagine herself as an (impartial) observer who does not know which person she will be. That is, the observer faces not only the real lottery $\ell$ over the social outcomes in $\mathcal{X}$, but also a hypothetical lottery $z$ over which identity in $\mathcal{I}$ she will assume. In forming preferences $\succsim$ over all such 'extended lotteries', the observer is forced to make interpersonal comparisons; for example, she is forced to compare being person $i$ in social state $x$ with being person $j$ in social state $x^{\prime}$.

Harsanyi assumed that when the observer imagines herself being person $i$ she adopts person $i$ 's preferences over the outcome lotteries. He also assumed that all individuals are expected utility maximizers, and that they continue to be so in the role of observer. Harsanyi argued that these "Bayesian rationality" axioms force the observer to be a utilitarian. More formally, over all extended lotteries $(z, \ell)$ in which the identity lottery and the outcome lotteries are independently distributed, the observer's preferences admit a representation of the form

$$
V(z, \ell)=\sum_{i} z_{i} U_{i}(\ell)
$$

where $z_{i}$ is the probability of assuming person $i$ 's identity and $U_{i}(\ell):=\int_{\mathcal{X}} u_{i}(x) \ell(d x)$ is person $i$ 's von Neumann-Morgenstern expected utility for the outcome lottery $\ell$. By fixing the identity lottery in (1) to be the equal-chance lottery $z^{u}=(1 / I, \ldots, 1 / I)$, the observer can use $V\left(z^{u}, \cdot\right)$ to obtain an 'impartial' ranking of the outcome lotteries (and hence, the associated social policies). ${ }^{1}$

\footnotetext{
1 This is essentially the method Weymark [18] uses to obtain an "impartial ranking" over social policies in his formalization of Harsanyi's impartial observer theorem. As an alternative, Gajdos and Kandil's [5] 'totally ignorant' observer considers all possible distributions over individuals to derive a ranking over social policies that is a weighted average of Harsanyi's utilitarian and Rawls' egalitarian criteria. Roemer [15] observes that such notions
} 
In our companion paper, we restricted attention to product lotteries, $\triangle(\mathcal{I}) \times \triangle(\mathcal{X})$, and, more significantly, only required each of Harsanyi's axioms to hold on a (further) restriction of this domain. In particular, we required the observer to satisfy a form of mixture independence only for mixtures of two product lotteries in which the outcome lottery was the same. In conjunction with a richness assumption, this yielded a generalized utilitarian representation of the form:

$$
V(z, \ell)=\sum_{i} z_{i} \phi_{i}\left(U_{i}(\ell)\right)
$$

where $z_{i}$ is again the probability of assuming person $i$ 's identity and $U_{i}(\ell)$ is again person $i$ 's expected utility from the outcome lottery $\ell$, but each $\phi_{i}($.$) is a (possibly non-linear) transformation$ of person $i$ 's expected utility. ${ }^{2}$ This allowed us to accommodate two objections that have been raised against Harsanyi's utilitarian impartial observer: one concerning ex ante fairness that is similar to Diamond's [3] critique of Harsanyi's [11] aggregation theorem; and one concerning different attitudes toward risk.

Fleurbaey [4] raises another objection to Harsanyi's utilitarianism which also applies to the generalized utilitarian representation in (2). To illustrate his objection, consider two individuals, $i$ and $j$ and three social outcomes $x_{i}, x_{j}$ and $\bar{x}$. Person $i$ strictly prefers outcome $x_{i}$ to outcome $x_{j}$ and is indifferent between $\bar{x}$ and a $(1 / 2,1 / 2)$ lottery over states $x_{i}$ or $x_{j}$ : that is, $u_{i}\left(x_{i}\right)>u_{i}\left(x_{j}\right)$ and $\frac{1}{2} u_{i}\left(x_{i}\right)+\frac{1}{2} u_{i}\left(x_{j}\right)=u_{i}(\bar{x})$. Person $j$ strictly prefers outcome $x_{j}$ to outcome $x_{i}$ and is also indifferent between $\bar{x}$ and a $(1 / 2,1 / 2)$ lottery over states $x_{i}$ or $x_{j}$ : that is, $u_{j}\left(x_{i}\right)<u_{j}\left(x_{j}\right)$ and $\frac{1}{2} u_{j}\left(x_{i}\right)+\frac{1}{2} u_{j}\left(x_{j}\right)=u_{j}(\bar{x})$. Perhaps, there is some (possibly indivisible) good, and $x_{i}$ is the state in which person $i$ gets the good; $x_{j}$ is the state in which person $j$ gets it, while $\bar{x}$ is a state in which neither receives the good but each gets some compromise alternative that each rates equivalent (in terms of her own risk preferences) to a lottery that gives her an equal chance of getting or not getting the good. Finally, suppose the observer is indifferent to: $(i)$ being $i$ getting $x_{i}$ and being $j$ getting $x_{j}$; (ii) being $i$ getting $\bar{x}$ and being $j$ getting $\bar{x}$; and, $(i i i)$ being $i$ getting $x_{j}$ and being

of impartiality reduce any consideration of social justice to simply one of rational prudence on the part of the observer. He, among others, contends that this constitutes an inadequate basis for a theory of social justice. We do not take a position on this here as the issues it raises are beyond the scope of this paper.

${ }^{2}$ See Grant et al [6], Theorem 1. Again by fixing the identity lottery to be the equal-chance lottery, the observer can use the representation in (2) to obtain an 'impartial' ranking of the outcome lotteries and the associated social policies. 
$j$ getting $x_{i}$.

Consider the two extended (product) lotteries illustrated in tables (a) and (b), in which rows are the people and columns are the outcomes.

\begin{tabular}{c|c|c|c|}
\multicolumn{1}{c}{$x_{i}$} & $\bar{x}$ & \multicolumn{1}{c}{$x_{j}$} \\
\cline { 2 - 4 }$i$ & $1 / 4$ & 0 & $1 / 4$ \\
\cline { 2 - 4 } & $1 / 4$ & 0 & $1 / 4$ \\
\hline & $1 / 4$ &
\end{tabular}

(a)

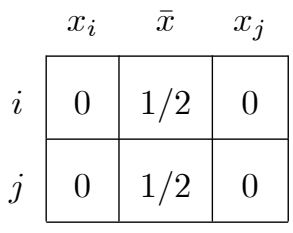

(b)

In each, the observer has an equal chance of being person $i$ or person $j$. In table (a), the observer has an equal chance of being in state $x_{i}$ or state $x_{j}$, but whichever of these two social states obtains, one person will get the good and the other will miss out. In table (b), state $\bar{x}$ obtains with probability 1 , that is, each individual receives the 'compromise' alternative. Given the assumptions we made in the previous paragraph for our generalized utilitarian observer, she must be indifferent between these two extended lotteries since by applying the generalized utilitarian representation in (2), we obtain:

$$
\begin{aligned}
V((\mathrm{a})) & =\frac{1}{2} \phi_{i}\left(\frac{1}{2} U_{i}\left(x_{i}\right)+\frac{1}{2} U_{i}\left(x_{j}\right)\right)+\frac{1}{2} \phi_{j}\left(\frac{1}{2} U_{j}\left(x_{i}\right)+\frac{1}{2} U_{j}\left(x_{j}\right)\right) \\
& =\frac{1}{2} \phi_{i}\left(U_{i}(\bar{x})\right)+\frac{1}{2} \phi_{j}\left(U_{j}(\bar{x})\right)=V((\mathrm{~b})) .
\end{aligned}
$$

Following Fleurbaey's reasoning, one might argue that an observer who is concerned about inequality should express a (strict) preference for the extended lottery (b) over (a), since the former results in no inequality ex post according to the interpersonal comparisons of the individuals' welfare implicit in the observer's preferences over extended lotteries, while the latter results in certain ex post inequality. In the setting of Harsanyi's [11] aggregation theorem, he proposes an alternative to generalized utilitarianism that he calls the 'expected equally-distributed equivalentutility maximizing.'

In our setting, the expected equally-distributed equivalent-utility representation takes the form:

$$
V(z, \ell)=\int \varphi^{-1}\left(\sum_{i} z_{i} \varphi\left(U_{i}(x)\right)\right) \ell(d x),
$$

where the function $\varphi(\cdot)$ plays a similar role as the $\phi_{i}(\cdot)$ functions in $(2)$, that is, translating each 
person $i$ 's von Neumann expected utility to the observer's expected utility for identity lotteries. Unlike the case of the generalized utilitarian, however, the observer's expected utility of the identity lottery $z$ for a given social state $x$ is translated back, using the inverse mapping $\varphi^{-1}$, so that it corresponds to the ex post utility level that if obtained for certain by every individual would be regarded by the observer as being equivalent to the distribution induced by $z$ of ex post welfares for that social state. The expected equally-distributed equivalent-utility is thus obtained by summing (more precisely, integrating) the probability weighted equally-distributed equivalent-(ex post) utilities. ${ }^{3}$

The main aim of this paper is to characterize, in the setting of Harsanyi's impartial observer theorem, the class of observers who admit a representation of the form in (3). We provide such an axiomatization in section 3 .

As its name suggests, the expected equally-distributed equivalent-utility representation bears a close resemblance to Atkinson's [2] equally-distributed equivalent income function for ranking income distributions and Rothschild-Stiglitz's [16] certainty-equivalent function for ranking lotteries over money/wealth. For these representations, the degree of concavity of the functions that are analogous to the function $\varphi$ in (3) measure the degree of aversion to income inequality and the degree of aversion to risk, respectively. We show in section 4 that the concavity of $\varphi$ corresponds to a notion of aversion to ex post inequality by the observer in the sense that a strict preference for the extended lottery described in table (b) over the extended lottery described in table (a) follows if $\varphi$ is concave. Furthermore, we show that the same axiom on the observer's preferences in Grant et al [6] that induced a generalized utilitarian observer to exhibit a concern about ex ante fairness is also sufficient (and necessary) for the $\varphi$ function in (3) to be concave. This provides an intriguing link between what it takes for a generalized utilitarian to exhibit an ex ante preference for fairness and for an expected equally-distributed equivalent-utility maximizer to display an aversion to ex post inequality.

\footnotetext{
3 Analogous to what Weymark (1991) showed can be done with the utilitarian representation, by fixing the identity lottery to be the equal-chance lottery, the observer can use the representation in (3) to obtain an 'impartial' ranking of the outcome lotteries and the associated social policies. A different approach was taken by Karni and Weymark (1998), whose domain consists of equal-chance identity lotteries only (but different individuals may face different social alternative lotteries). As this domain does not include identity lotteries in which the observer can be a given individual with certainty, Karni and Weymark had to strengthen Harsanyi's principle of acceptance.
} 
Finally, in section 5, we consider an observer who satisfies both sets of axioms (including both independence properties) that characterize the generalized utilitarian and the expected equallydistributed equivalent-utility maximizer. A natural conjecture is that this would be enough to induce utilitarianism. It turns out that this is not quite enough, and we illustrate the gap with an example. However, if there are three or more agents, then under some mild richness conditions on the preferences, it is indeed only utilitarians who reside in the intersection of the set of generalized utilitarians and the set of expected equally-distributed equivalent-utility maximizers. This provides a new axiomatization of utilitarianism.

\section{Set up and Notation}

Let society consist of a finite set of individuals $\mathcal{I}=\{1, \ldots, n\}, n \geq 2$, with generic elements $i$ and $j$. The set of final outcomes or social states is denoted by $\mathcal{X}$ with generic element $x$. The set $\mathcal{X}$ is assumed to have more than one element and to be a compact metrizable space and associated with it is the set of events $\mathcal{E}$, which is taken to be the Borel sigma-algebra of $\mathcal{X}$. Let $\triangle(\mathcal{X})$ (with generic element $\ell$ ) denote the set of outcome lotteries; that is, the set of probability measures on $(\mathcal{X}, \mathcal{E})$ endowed with the weak convergence topology. With slight abuse of notation, we will let $x$

or sometimes $[x]$ denote the degenerate outcome lottery that assigns probability weight 1 to social state $x$.

Each individual $i$ in $\mathcal{I}$ is endowed with a preference relation $\succsim_{i}$ defined over the set of outcome lotteries $\triangle(\mathcal{X})$. We assume throughout that for each $i$ in $\mathcal{I}$, the preference relation $\succsim_{i}$ is a complete, transitive binary relation on $\triangle(\mathcal{X})$, and that its asymmetric part $\succ_{i}$ is non-empty. We assume these preferences are continuous in that weak upper and weak lower contour sets are closed. Hence, for each $\succsim_{i}$ there exists a non-constant continuous function $V_{i}: \triangle(\mathcal{X}) \rightarrow \mathbb{R}$, satisfying for any $\ell$ and $\ell^{\prime}$ in $\triangle(\mathcal{X}), V_{i}(\ell) \geq V_{i}\left(\ell^{\prime}\right)$ if and only if $\ell \succsim_{i} \ell^{\prime}$. In summary, a society may be characterized by the tuple $\left\langle\mathcal{X}, \mathcal{I},\left\{\succsim_{i}\right\}_{i \in \mathcal{I}}\right\rangle$.

In Harsanyi's story, an observer imagines herself behind a veil of ignorance, uncertain about which identity she will assume in the given society. Let $\triangle(\mathcal{I})$ denote the set of identity lotteries on $\mathcal{I}$. Let $z$ denote the typical element of $\triangle(\mathcal{I})$, and let $z_{i}$ denote the probability assigned by the 
identity lottery $z$ to individual $i$. They represent the imaginary risks in the mind of the observer as she conducts thought experiments in which she envisages assuming the identity of someone else. With slight abuse of notation, we will let $i$ or sometimes $[i]$ denote the degenerate identity lottery that assigns probability weight 1 to the observer assuming the identity of individual $i$.

As discussed above, we assume that the outcome and identity lotteries faced by the observer are independently distributed; that is, she faces a product lottery $(z, \ell) \in \triangle(\mathcal{I}) \times \triangle(\mathcal{X})$. We refer to this as an identity-outcome lottery or, where no confusion will arise, simply as a product lottery.

The observer is endowed with a preference relation $\succsim$ defined over $\triangle(\mathcal{I}) \times \triangle(\mathcal{X})$. We assume throughout that $\succsim$ is complete, transitive continuous (in that weak upper and weak lower contour sets are closed in the product topology), and that its asymmetric part $\succ$ is non-empty, and so it admits a (non-trivial) continuous representation $V: \triangle(\mathcal{I}) \times \triangle(\mathcal{X}) \rightarrow \mathbb{R}$. That is, for any pair of product lotteries, $(z, \ell)$ and $\left(z^{\prime}, \ell^{\prime}\right),(z, \ell) \succsim\left(z^{\prime}, \ell^{\prime}\right)$ if and only if $V(z, \ell) \geq V\left(z^{\prime}, \ell^{\prime}\right)$.

As we noted in the introduction above, $\succsim$ and its representation $V(\cdot, \cdot)$ explicitly involve interpersonal comparisons. For example, $V(i, x)>V\left(j, x^{\prime}\right)$ means the observer assesses that it is better to be person $i$ in social state $x$ than person $j$ in social state $x^{\prime}$. Hence, for each $x$, we can view the vector $(V(1, x), \ldots, V(n, x))$ as the distribution across individuals of interpersonallycomparable utilities associated with that social state. ${ }^{4}$

As discussed in the introduction, we have the following special families of preferences.

Utilitarianism The observer is a utilitarian if her preferences $\succsim$ admit a representation of the form

$$
V(z, \ell)=\sum_{i=1}^{n} z_{i} U_{i}(\ell),
$$

where, for each individual $i$ in $\mathcal{I}, U_{i}: \triangle(\mathcal{X}) \rightarrow \mathbb{R}$ is a von Neumann-Morgenstern expectedutility representation of $\succsim_{i}$; i.e., $U_{i}(\ell):=\int_{\mathcal{X}} U_{i}(x) \ell(d x)$.

(Ex Ante) Generalized Utilitarianism The observer is an (ex ante) generalized utilitarian if

\footnotetext{
${ }^{4}$ In Fleurbaey [4], social states are not explicitly modelled. Rather, there are 'states of the world' with given probabilities and the data are state-contingent distributions of ex post utilities across individuals. The utilities are assumed to be interpersonally comparable.
} 
her preferences $\succsim$ admit a representation $\left\langle\left\{U_{i}, \phi_{i}\right\}_{i \in \mathcal{I}}\right\rangle$ of the form

$$
V(z, \ell)=\sum_{i=1}^{n} z_{i} \phi_{i}\left(U_{i}(\ell)\right),
$$

where, for each individual $i$ in $\mathcal{I}, \phi_{i}: \mathbb{R} \rightarrow \mathbb{R}$ is a continuous, increasing function, and $U_{i}: \triangle(\mathcal{X}) \rightarrow \mathbb{R}$ is a von Neumann-Morgenstern expected-utility representation of $\succsim_{i}$.

Also discussed in the introduction is the following representation for an observer that can exhibit Fleurbaey's [4] notion of aversion to ex post inequality.

Expected Equally-Distributed Equivalent Utility The observer is an expected equally-distributed equivalent-utility maximizer if her preferences $\succsim$ admit a representation $\left\langle\left\{U_{i}\right\}_{i \in \mathcal{I}}, \varphi\right\rangle$ of the form

$$
V(z, \ell)=\int_{\mathcal{X}} \varphi^{-1}\left(\sum_{i=1}^{n} z_{i} \varphi\left(U_{i}(x)\right)\right) \ell(d x),
$$

where, $\varphi: \mathbb{R} \rightarrow \mathbb{R}$ is a continuous, increasing function, and for each individual $i$ in $\mathcal{I}$, $U_{i}: \triangle(\mathcal{X}) \rightarrow \mathbb{R}$ is a von Neumann-Morgenstern expected-utility representation of $\succsim_{i}$.

\section{$3 \quad$ Expected Equally-Distributed Equivalent-Utility}

In this section, we provide an axiomatization of an expected equally-distributed equivalent-utility maximizer.

The first axiom is Harsanyi's acceptance principle. For degenerate product lotteries of the form $(i, \ell)$ or $\left(i, \ell^{\prime}\right)$, the observer knows she will assume identity $i$ for sure. The acceptance principle requires that, in this case, the observer's preferences $\succsim$ must coincide with that individual's preferences $\succsim_{i}$ over outcome lotteries.

Acceptance Principle. For all $i$ in $\mathcal{I}$ and all $\ell, \ell^{\prime} \in \triangle(\mathcal{X}), \ell \succsim_{i} \ell^{\prime}$ if and only if $(i, \ell) \succsim\left(i, \ell^{\prime}\right)$.

Second, we assume that the observer's preferences satisfy independence for certain mixtures of product lotteries. Note we need to be careful. The set of product lotteries $\triangle(\mathcal{I}) \times \triangle(\mathcal{X})$ is not a convex subset of $\triangle(\mathcal{I} \times \mathcal{X})$ and hence not all probability mixtures of product lotteries are well defined. We first define an independence property that is a restriction of the one introduced in Grant et al [6]. 
Independence over Identity Lotteries with Degenerate Outcome Lotteries. Suppose $(z, x)$, $\left(z^{\prime}, x^{\prime}\right) \in \triangle(\mathcal{I}) \times \mathcal{X}$ are such that $(z, x) \sim\left(z^{\prime}, x^{\prime}\right)$. Then, for all $\tilde{z}, \tilde{z}^{\prime} \in \triangle(\mathcal{I}):(\tilde{z}, x) \succsim$ $\left(\tilde{z}^{\prime}, x^{\prime}\right)$ if and only if $(\alpha \tilde{z}+(1-\alpha) z, x) \succsim\left(\alpha \tilde{z}^{\prime}+(1-\alpha) z^{\prime}, x^{\prime}\right)$ for all $\alpha$ in $(0,1]$.

To understand the mechanics of this axiom, first notice that the two mixtures on the right side of the implication are identical to $\alpha(\tilde{z}, x)+(1-\alpha)(z, x)$ and $\alpha\left(\tilde{z}^{\prime}, x^{\prime}\right)+(1-\alpha)\left(z^{\prime}, x^{\prime}\right)$, respectively. These two mixtures of product lotteries are well defined: they mix identity lotteries holding the social state (that is, degenerate outcome lottery) fixed. ${ }^{5}$ Second, notice that the two product lotteries, $(z, x)$ and $\left(z^{\prime}, x^{\prime}\right)$, that are 'mixed in' with weight $(1-\alpha)$ are themselves indifferent. The axiom states that 'mixing in' two indifferent lotteries (with equal weight) preserves the original preference between $(\tilde{z}, x)$ and $\left(\tilde{z}^{\prime}, x^{\prime}\right)$ prior to mixing.

As the product lotteries being mixed hold the social state fixed, there is no uncertainty about each individual's ex post utility. Thus, the product lotteries being mixed are essentially equivalent from the perspective of an ex post egalitarian. The only uncertainty that is to be resolved concerns which particular identity the observer will assume. As the domain of this randomization is purely hypothetical, coupled with the fact that the ex post utilities of the individuals are already determined, standard arguments made against independence with regard to fairness or inequality aversion considerations do not seem to apply, so the axiom postulates that independence over identity lotteries holds in this restricted (but arguably, intuitive) domain.

We next consider a 'dual' independence property that holds for mixtures of outcome lotteries holding the identity lotteries fixed. ${ }^{6}$

Independence over Outcome Lotteries (for the Observer). Suppose $(z, \ell),\left(z^{\prime}, \ell^{\prime}\right) \in \triangle(\mathcal{I}) \times$ $\triangle(\mathcal{X})$ are such that $(z, \ell) \sim\left(z^{\prime}, \ell^{\prime}\right)$. Then, for all $\tilde{\ell}, \tilde{\ell}^{\prime} \in \triangle(\mathcal{X}):(z, \tilde{\ell}) \succsim\left(z^{\prime}, \tilde{\ell}^{\prime}\right)$ if and only if $(z, \alpha \tilde{\ell}+(1-a) \ell) \succsim\left(z^{\prime}, \alpha \tilde{\ell}^{\prime}+(1-a) \ell^{\prime}\right)$ for all $\alpha$ in $(0,1]$.

Now the product lotteries being mixed hold the identity lottery fixed, so the mixing concerns

\footnotetext{
${ }^{5}$ In Grant et al [6], the axiom applies holding fixed any outcome lottery, not just a degenerate outcome lottery as is done here.

${ }^{6}$ More properly speaking, this axiom is dual to the independence over identity lotteries that was the key axiom in the characterization of the generalized utilitarian impartial observer in Grant et al [6].
} 
the uncertainty associated with which social outcome will obtain. Thus, the axiom essentially requires that if the identity lottery is fixed, then the observer should exhibit an independence property analogous to what is required from an individual whose preferences over outcome lotteries are expected utility. Indeed, if the observer satisfies acceptance, then this independence entails that the preferences of each individual satisfy the standard independence of expected utility:

Independence over Outcome Lotteries (for Individual $i$ ). For all $\ell$, $\ell^{\prime}$ and $\ell^{\prime \prime}$ in $\triangle(\mathcal{X})$, $\ell \succsim_{i} \ell^{\prime}$ if and only if $\alpha \ell+(1-\alpha) \ell^{\prime \prime} \succsim_{i} \alpha \ell^{\prime}+(1-\alpha) \ell^{\prime \prime}$ for all $\alpha$ in $(0,1]$.

Hence, we have the individuals are expected utility maximizers and thus each $\succsim_{i}$ admits a von Neumann-Mogernstern representation.

Proposition 1 Suppose that $\succsim$ satisfies acceptance and independence over outcome lotteries, then for all $i$ in $\mathcal{I}, \succsim_{i}$ satisfies independence over outcome lotteries.

Proof. Fix $\ell, \ell^{\prime}$ and $\ell^{\prime \prime}$ in $\triangle(\mathcal{X})$ and $\alpha$ in $(0,1]$. By acceptance, $\ell \succsim_{i} \ell^{\prime}$ if and only if $(i, \ell) \succsim\left(i, \ell^{\prime}\right)$ and $\alpha \ell+(1-\alpha) \ell^{\prime \prime} \succsim_{i} \alpha \ell^{\prime}+(1-\alpha) \ell^{\prime \prime}$ if and only if $\left(i, \alpha \ell+(1-\alpha) \ell^{\prime \prime}\right) \succsim$ $\left(i, \alpha \ell^{\prime}+(1-\alpha) \ell^{\prime \prime}\right)$. But applying independence over outcome lotteries, we have $(i, \ell) \succsim\left(i, \ell^{\prime}\right)$ if and only if $\left(i, \alpha \ell+(1-\alpha) \ell^{\prime \prime}\right) \succsim\left(i, \alpha \ell^{\prime}+(1-\alpha) \ell^{\prime \prime}\right)$. Hence, $\ell \succsim_{i} \ell^{\prime}$ if and only if $\alpha \ell+(1-\alpha) \ell^{\prime \prime} \succsim_{i}$ $\alpha \ell^{\prime}+(1-\alpha) \ell^{\prime \prime}$. Since $\alpha$ can be any value in $(0,1]$, the result holds.

To obtain our representation result, we work with two richness conditions on the domain of individual preferences. The first entails that none of the outcomes under consideration are Pareto dominated. The second entails that there are no 'dominated' identity lotteries.

Absence of Unanimity over Outcomes For all $x, x^{\prime} \in \mathcal{X}$, if $x \succ_{i} x^{\prime}$ for some $i$ in $\mathcal{I}$, then there exists $j$ in $\mathcal{I}$ such that $x^{\prime} \succ_{j} x$.

Redistributive Scope For all $z, z^{\prime}$ in $\triangle(\mathcal{I})$, if $(z, x) \succ\left(z^{\prime}, x\right)$ for some $x$ in $\mathcal{X}$, then there exists $x^{\prime}$ in $\mathcal{X}$ such that $\left(z^{\prime}, x^{\prime}\right) \succ\left(z, x^{\prime}\right)$. 
Absence of Unanimity over Outcomes is perhaps a natural restriction in the context of Harsanyi's thought experiment. The whole exercise is motivated by the need to make social choices when agents disagree. We do not need to imagine ourselves as an observer facing an identity lottery to rule out an outcome that every individual agrees is dominated by some other outcome, that is, that is Pareto dominated. We contend that this should still hold even if the observer may have concerns about ex post inequality. In fact, in his setting, Fleurbaey [4] explicitly requires that social preference over constant acts respect the Pareto ordering. ${ }^{7}$

Redistributive scope, on the other hand, rules out the case where one individual is always worse off than another (from the perspective of the observer) regardless of the outcome. There are many economic contexts in which this condition will be met. ${ }^{8}$

These axioms are sufficient to yield an expected equally-distributed equivalent-utility representation.

Theorem 2 Suppose that absence of unanimity over outcomes and redistributive scope apply. Then, the observer satisfies acceptance, independence over identity lotteries with degenerate outcome lotteries and independence over outcome lotteries if and only if her preferences $\succsim$ admit a representation of the form

$$
V(z, \ell)=\int_{\mathcal{X}} \varphi^{-1}\left(\sum_{i} z_{i} \varphi\left(U_{i}(x)\right)\right) \ell(d x)
$$

where, for each individual $i$ in $\mathcal{I}, U_{i}: \triangle(\mathcal{X}) \rightarrow \mathbb{R}$ is a von Neumann-Morgenstern expectedutility representation of $\succsim_{i} ;$ and $\varphi: \mathbb{R} \rightarrow \mathbb{R}$ is a continuous, increasing function. Moreover, the composite functions $\varphi \circ U_{i}$ are unique up to common affine transformations.

The proof is in the appendix but we provide here a sketch. We begin by noting that as a corollary to a result from another companion paper (Grant et al, [7]), it follows that in the presence of absence of unanimity over outcomes, acceptance and independence over identity lotteries with degenerate outcome lotteries yields a generalized utilitarian representation restricted to $\triangle(\mathcal{I}) \times$

\footnotetext{
7 In Grant et al [6], the observer was not concerned with ex post inequality per se, so we required absence of unanimity to hold for all outcome lotteries, not just outcomes (that is, degenerate outcome lotteries).

${ }^{8}$ However, there are other contexts in which the condition will fail. For example, in The House at Pooh Corner, readers might prefer to be Tigger than to be Eyeore regardless of the outcome (Milne, [13]).
} 
$\mathcal{X}$ of the form:

$$
\hat{V}(z, x)=\sum_{i} z_{i} W_{i}(x)
$$

By adding 'outcome' independence, it follows that all the functions $W_{i}$ are common monotonic transformations of each individual's expected utility function $U_{i}$. This in turn allows us to express a version of the representation given in (4) in which $W_{i}(x)=\varphi \circ U_{i}(x)$. Finally by extending this to all product lotteries in $\triangle(\mathcal{I}) \times \triangle(\mathcal{X})$, we obtain the the expected equally-distributed equivalent-utility representation in (3).

\section{Ex Post Egalitarianism}

The only restrictions placed by the axioms in Theorem 2 on the shape of the function $\varphi$ from the expected equally-distributed equivalent-utility representation are that it is continuous and increasing. In a standard utilitarian social welfare function, each $U_{i}$-function maps individual $i$ 's income to an individual utility. These incomes differ across people, and concavity of the $U_{i}$-functions is associated with egalitarianism over incomes. In the expected equally-distributed equivalent-utility representation, the function $\varphi$ maps individual $i$ 's ex post utility $U_{i}(x)$ to the ex post utility of the observer. These ex post utilities may differ across people, and concavity of the $\varphi$-functions is associated with egalitarianism over ex post utilities, often called ex post egalitarianism. ${ }^{9}$ To see the relevance of this for Fleurbaey's notion of ex post inequality aversion, recall from the introduction the two identity-outcome lotteries presented in tables (a) and (b), which correspond to

$$
\left(\frac{1}{2}[i]+\frac{1}{2}[j], \frac{1}{2}\left[x_{i}\right]+\frac{1}{2}\left[x_{j}\right]\right) \text { and }\left(\frac{1}{2}[i]+\frac{1}{2}[j], \bar{x}\right)
$$

respectively. Recall also, each individual is indifferent between the outcome lottery $\frac{1}{2}\left[x_{i}\right]+\frac{1}{2}\left[x_{j}\right]$ and the (degenerate) outcome lottery $\bar{x}$, and the observer is indifferent between: (i) being $i$ getting $x_{i}$ and being $j$ getting $x_{j}$; (ii) being $i$ getting $\bar{x}$ and being $j$ getting $\bar{x}$; and, (iii) being $i$ getting $x_{j}$ and being $j$ getting $x_{i}$. Following Fleurbaey's line of argument, this implies that an ex post

\footnotetext{
${ }^{9}$ See, for example, Harel, Safra and Segal [8] and Fleurbaey [4].
} 
egalitarian should express the strict preference

$$
\left(\frac{1}{2}[i]+\frac{1}{2}[j], \bar{x}\right) \succ\left(\frac{1}{2}[i]+\frac{1}{2}[j], \frac{1}{2}\left[x_{i}\right]+\frac{1}{2}\left[x_{j}\right]\right),
$$

since the former identity-outcome lottery results in no inequality ex post, while the latter results in certain ex post inequality.

To see how a strictly concave $\varphi$ leads to our expected equally-distributed equivalent-utility maximizing observer exhibiting the desired strict preference expressed in (5), first notice that $V\left(i, x_{i}\right)=V\left(j, x_{j}\right)$ implies that $\varphi^{-1} \circ \varphi\left(U_{i}\left(x_{i}\right)\right)=\varphi^{-1} \circ \varphi\left(U_{j}\left(x_{j}\right)\right)$, that is, $U_{i}\left(x_{i}\right)=U_{j}\left(x_{j}\right)$ whether or not $\varphi$ is concave. Similarly, $V(i, \bar{x})=V(j, \bar{x})$ and $V\left(i, x_{j}\right)=V\left(j, x_{i}\right)$ imply that $U_{i}(\bar{x})=U_{j}(\bar{x})$ and $U_{i}\left(x_{j}\right)=U_{j}\left(x_{i}\right)$, respectively.

With a strictly concave $\varphi$, however, the observer will express the strict preference:

$$
\left(i, \frac{1}{2}\left[x_{i}\right]+\frac{1}{2}\left[x_{j}\right]\right) \succ\left(\frac{1}{2}[i]+\frac{1}{2}[j], x_{i}\right) .
$$

This follows since

$$
\begin{aligned}
& V\left(\frac{1}{2}[i]+\frac{1}{2}[j], x_{i}\right)=\varphi^{-1} \circ\left(\frac{1}{2} \varphi\left(U_{i}\left(x_{i}\right)\right)+\frac{1}{2} \varphi\left(U_{j}\left(x_{i}\right)\right)\right) \\
< & \varphi^{-1} \circ \varphi\left(\frac{1}{2} U_{i}\left(x_{i}\right)+\frac{1}{2} U_{j}\left(x_{i}\right)\right),\left(\text { since } \varphi \text { is strictly concave and } U_{i}\left(x_{i}\right)>U_{j}\left(x_{i}\right)\right) \\
= & \frac{1}{2} U_{i}\left(x_{i}\right)+\frac{1}{2} U_{i}\left(x_{j}\right)=V\left(i, \frac{1}{2}\left[x_{i}\right]+\frac{1}{2}\left[x_{j}\right]\right) .
\end{aligned}
$$

Notice that

$$
\begin{gathered}
V\left(\frac{1}{2}[i]+\frac{1}{2}[j], \bar{x}\right)=\varphi^{-1} \circ\left(\frac{1}{2} \varphi\left(U_{i}(\bar{x})\right)+\frac{1}{2} \varphi\left(U_{j}(\bar{x})\right)\right) \\
=\varphi^{-1} \circ \varphi\left(U_{i}(\bar{x})\right)=\frac{1}{2} U_{i}\left(x_{i}\right)+\frac{1}{2} U_{i}\left(x_{j}\right)=V\left(i, \frac{1}{2}\left[x_{i}\right]+\frac{1}{2}\left[x_{j}\right]\right),
\end{gathered}
$$

where the second equality follows from the assumption that $U_{i}(\bar{x})=U_{j}(\bar{x})$. Hence, we have,

$$
\begin{aligned}
& V\left(\frac{1}{2}[i]+\frac{1}{2}[j], \frac{1}{2}\left[x_{i}\right]+\frac{1}{2}\left[x_{j}\right]\right) \\
= & \frac{1}{2} \varphi^{-1} \circ\left(\frac{1}{2} \varphi\left(U_{i}\left(x_{i}\right)\right)+\frac{1}{2} \varphi\left(U_{j}\left(x_{i}\right)\right)\right)+\frac{1}{2} \varphi^{-1} \circ\left(\frac{1}{2} \varphi\left(U_{i}\left(x_{j}\right)\right)+\frac{1}{2} \varphi\left(U_{j}\left(x_{j}\right)\right)\right) \\
= & \varphi^{-1} \circ\left(\frac{1}{2} \varphi\left(U_{i}\left(x_{i}\right)\right)+\frac{1}{2} \varphi\left(U_{j}\left(x_{i}\right)\right)\right)\left[\text { since } U_{i}\left(x_{j}\right)=U_{j}\left(x_{i}\right) \& U_{j}\left(x_{j}\right)=U_{i}\left(x_{i}\right)\right] \\
= & V\left(\frac{1}{2}[i]+\frac{1}{2}[j], x_{i}\right) .
\end{aligned}
$$


Combining these last two sets of inequalities with (6) implies the strict preference in (5).

We will show that concavity of the function $\varphi$ is equivalent to an axiom that we introduced in Grant et al [6] to enable a generalized utilitarian observer to exhibit a concern about (ex ante) fairness that is similar to Diamond's [3] critique of Harsanyi's aggregation theorem. In the discussion above, we showed that a (strictly) concave $\varphi$ led the expected equally-distributed equivalent-utility maximizing observer to exhibit the preference in (6). The axiom we propose may be viewed as a generalization of this expression of preference. To explain the axiom, suppose the observer is indifferent between the identity-outcome lotteries $\left(z, \ell^{\prime}\right)$ and $\left(z^{\prime}, \ell\right)$. Consider now a product lottery $(z, \ell)$ that (in general) lies in a different indifference set. There are two ways to randomize between these indifference sets while remaining in the set of product lotteries. The product lottery $\left(z, \alpha \ell+(1-\alpha) \ell^{\prime}\right)$ randomizes between these indifference sets in outcome lotteries (i.e., real life chances); while the product lottery $\left(\alpha z+(1-\alpha) z^{\prime}, \ell\right)$ randomizes between these indifference sets in identity lotteries (i.e., imaginary accidents of birth). We argued in Grant et al [6] that a preference for fairness corresponds to preferring a randomization between these indifference sets in outcome lotteries (i.e., real life chances) to a randomization in identity lotteries (i.e., imaginary accidents of birth).

Preference for Life Chances. For any pair of identity lotteries $z$ and $z^{\prime}$ in $\triangle(\mathcal{I})$ and any pair of outcome lotteries $\ell$ and $\ell^{\prime}$ in $\triangle(\mathcal{X})$, if $\left(z, \ell^{\prime}\right) \sim\left(z^{\prime}, \ell\right)$, then $\left(z, \alpha \ell+(1-\alpha) \ell^{\prime}\right) \succsim$ $\left(\alpha z+(1-\alpha) z^{\prime}, \ell\right)$ for all $\alpha$ in $(0,1)$.

Adding this axiom to the conditions of Theorem 2 yields the desired property for $\varphi$.

Proposition 3 (Concavity) Suppose that absence of unanimity over outcomes and redistributive scope apply. Then, an expected equally-distributed equivalent-utility maximizing observer with representation $\left\langle\left\{U_{i}\right\}_{i \in \mathcal{I}}, \varphi\right\rangle$ exhibits preference for life chances if and only if $\varphi$ is concave.

Notice if we strengthened the axiom Preference for Life Chances so that $\left(\alpha z+(1-\alpha) z^{\prime}, \ell\right) \succsim$ $\left(z, \alpha \ell+(1-\alpha) \ell^{\prime}\right)$ held as well, or equivalently we required the observer to be indifferent between these two identity-outcome lotteries, then the corresponding proposition would require $\varphi$ to be 
affine. And by taking an appropriate normalization, we see that such an observer's preferences are utilitarian. In the next section, we consider an alternative way the axioms can be strengthened to induce (full) utilitarianism.

\section{Independence along both margins and Utilitarianism.}

In Grant et al [6], we assumed the observer's preferences satisfied the strengthening of Absence of Unanimity over Outcomes that requires none of the outcome lotteries under consideration are Pareto dominated.

Absence of Unanimity For all $\ell, \ell^{\prime} \in \Delta(\mathcal{X})$, if $\ell \succ_{i} \ell^{\prime}$ for some $i$ in $\mathcal{I}$, then there exists $j$ in $\mathcal{I}$ such that $\ell^{\prime} \succ_{j} \ell$.

Given preferences that satisfy absence of unanimity, we showed in Theorem 1 of Grant et al [6] that acceptance, independence over outcome lotteries for each individual's preferences and the following independence property characterized ex ante generalized utilitarianism.

Independence over Identity Lotteries. Suppose $(z, \ell),\left(z^{\prime}, \ell^{\prime}\right) \in \triangle(\mathcal{I}) \times \triangle(\mathcal{X})$ are such that $(z, \ell) \sim\left(z^{\prime}, \ell^{\prime}\right)$. Then, for all $\tilde{z}, \tilde{z}^{\prime} \in \triangle(\mathcal{I}):(\tilde{z}, \ell) \succsim\left(\tilde{z}^{\prime}, \ell^{\prime}\right)$ if and only if $(\alpha \tilde{z}+(1-\alpha) z, \ell) \succsim$ $\left(\alpha \tilde{z}^{\prime}+(1-\alpha) z^{\prime}, \ell^{\prime}\right)$ for all $\alpha$ in $(0,1]$.

A natural question is whether (given the richness conditions, redistributive scope and absence of unanimity) acceptance, independence over identity lotteries and independence over outcome lotteries are enough to induce utilitarianism. An observer might satisfy both independences because she views the two types of randomization symmetrically - if independence applies to one margin, then perhaps it should apply to the other - without taking a direct position on whether the two types of randomization are equivalent.

It turns out, however, that acceptance and both of these independence properties are not enough to induce utilitarianism. In fact, we can see this with a simple example similar to the one that appeared in the introduction. Once again, suppose that there are two individuals, $i$ and $j$, and two states, $x_{i}$ and $x_{j}$, denoting which agent is given a (possibly indivisible) good. Suppose 
that the observer's preferences again satisfy $\left(i, x_{i}\right) \sim\left(j, x_{j}\right)$ and $\left(i, x_{j}\right) \sim\left(j, x_{i}\right)$. Suppose that both individuals satisfy independence. Specifically, for any outcome lottery $\ell$, player $i$ 's expected utility is given by $U_{i}(\ell)=\ell\left(x_{i}\right)-\ell\left(x_{j}\right)$ and player $j$ 's expected utility is given by $U_{j}(\ell)=\ell\left(x_{j}\right)-$ $\ell\left(x_{i}\right)$. Hence, $U_{i}\left(x_{i}\right)=U_{j}\left(x_{j}\right)=1$ and $U_{i}\left(x_{j}\right)=U_{j}\left(x_{i}\right)=-1$. Let the observer's preferences be given by the expected equally-distributed equivalent-utility representation

$$
V(z, \ell):=\ell\left(x_{i}\right) \varphi^{-1}\left(z_{i}-z_{j}\right)+\ell\left(x_{j}\right) \varphi^{-1}\left(z_{j}-z_{i}\right),
$$

where the function $\varphi$ is given by:

$$
\varphi(u)=\left\{\begin{array}{ll}
u^{m} & \text { for } u \geq 0 \\
-(-u)^{m} & \text { for } u<0
\end{array} \quad \text {, for some } m>0 .\right.
$$

By Theorem 2, it follows the observer's preferences satisfy acceptance, independence over outcome lotteries and independence over identity lotteries with degenerate outcome lotteries. It is less obvious that they satisfy (full) independence over identity lotteries, but this can be readily seen by considering the monotonic transformation

$$
\begin{gathered}
\hat{V}(z, \ell):=\quad \varphi \circ V(z, \ell)=\varphi \circ\left(\left[\ell\left(x_{i}\right)-\ell\left(x_{j}\right)\right] \varphi^{-1}\left(z_{i}-z_{j}\right)\right) \\
=\quad z_{i} \varphi\left(U_{i}(\ell)\right)+z_{j} \varphi\left(U_{j}(\ell)\right) .
\end{gathered}
$$

$\hat{V}$ is a generalized utilitarian representation. Hence, it follows from Grant et al $[6]$ that the preferences satisfy independence over identity lotteries as well.

These preferences even have the property (similar to utilitarianism) that if the observer thinks she is equally likely to be either person, then she is indifferent as to who gets the good. But these preferences do not satisfy utilitarianism unless $m=1$. To see this, notice that these preferences fail to exhibit indifference between life chances and accidents of birth. For example, we have $\left(i, x_{i}\right) \sim\left(j, x_{j}\right)$, but $\left(i, \alpha x_{i}+(1-\alpha) x_{j}\right) \nsim\left(\alpha[i]+(1-\alpha)[j], x_{i}\right)$ except in the special case when $\alpha=\frac{1}{2}$.

Nevertheless, the intuition that independence along both margins should imply utilitarianism is correct. If there are three or more agents, then under some mild richness conditions on the preferences, the combination of identity independence, outcome independence and acceptance do imply utilitarianism. 
The example shows that the two richness conditions we have used so far, redistributive scope and absence of unanimity, are not enough. But they are close. The $\varphi$-function in the example is a homogenous function. Again, this is general: Lemma 9 in the appendix shows that if we start from a generalized utilitarian representation with a common $\phi$-function, then independence over outcome lotteries implies that this common $\phi$-function is always homogenous; that is homogeneity is necessary. But homogeneity is not sufficient except in very special cases. Notice in the example that the point of inflection of the homogenous $\varphi$-function (the "zero") occurs exactly at outcome lottery $\hat{\ell}$, where $\hat{\ell}\left(x_{i}\right)=1 / 2$. For this outcome lottery, the observer is indifferent over which identity lottery she faces. This is a very "knife-edge" property, and it can be ruled out in a number of ways. The following extra richness condition suffices.

Three-Player Richness For all outcomes $x, y$ in $\mathcal{X}$ and all $\alpha$ in $[0,1]$, there exist individuals $i$ and $j$ in $\mathcal{I}$ such that $(i, \alpha[x]+(1-\alpha)[y]) \succ(j, \alpha[x]+(1-\alpha)[y])$.

Given redistributive scope, three-player richness implies that there must be at least three individuals. In words, it says that there is no outcome lottery involving just two outcomes at which the observer is indifferent over all the possible identities she could assume. In the example above, the condition was violated at $\hat{\ell}$, where $\hat{\ell}\left(x_{i}\right)=1 / 2$, since the observer was indifferent between being either person there. If we add a third person $k$ to the example, then the condition would be met provided that either the outcome lottery at which the observer is indifferent between being person $i$ or person $k$ or the outcome lottery at which she is indifferent between being person $j$ or person $k$ is not exactly equal to $1 / 2 .^{10}$

With this extra condition in place (and hence troublesome examples like the example above ruled out), the symmetric richness conditions and symmetric independence axioms over identity and outcome lotteries yield Harsanyi's utilitarianism.

Theorem 4 (Utilitarianism) Suppose that absence of unanimity, redistributive scope and threeplayer richness all apply. Then, the following are equivalent:

\footnotetext{
10 Notice that if there are three or more possible outcomes, the condition still only places restrictions for lotteries involving just two. In particular, there still could be some lottery in the interior of the simplex where the observer is indifferent as to identity. The condition would, however, be violated if there were divisible and disposable private goods and (hence) some outcome that equalized the welfare of all individuals. In that setting, however, since the outcome set is itself very rich, we can anyway induce a sufficiently rich set of utility lotteries.
} 
(a) The observer satisfies the acceptance principle, independence over identity lotteries and independence over outcome lotteries.

(b) There exist a continuous function $V: \triangle(\mathcal{I}) \times \triangle(\mathcal{X}) \rightarrow \mathbb{R}$ that represents $\succsim$ and, for each $i$ in $\mathcal{I}$, a function $U_{i}: \triangle(\mathcal{X}) \rightarrow \mathbb{R}$ that is a von Neumann-Morgenstern expected-utility representation of $\succsim_{i}$ such that for all $(z, \ell)$ in $\triangle(\mathcal{I}) \times \triangle(\mathcal{X})$,

$$
V(z, \ell)=\int_{\mathcal{X}}\left(\sum_{i=1}^{I} z_{i} U_{i}(x)\right) \ell(d x) .
$$

Moreover the functions $U_{i}$ are unique up to common affine transformation.

The proof is in the appendix. The example discussed above shows that three-player richness is essential.

\section{Appendix: Proofs}

Proof of Theorem 2 (Ex Post Generalized Utilitarianism): It is immediate that the preferences generated by the representation satisfy the axioms. We will show that the axioms imply the representation

Step 1. Generalized Utilitarian Representation of $\succsim$ restricted to $\triangle(\mathcal{I}) \times \mathcal{X}$. The following lemma is a corollary of Theorem 1 in Grant et al [7]. This Theorem establishes a representation of the preferences that is affine in identity lotteries. Since we only assume the independence over identity lotteries for identity-outcome lotteries that are degenerate in the outcome lottery, the affine representation need only hold for $\succsim$ restricted to $\triangle(\mathcal{I}) \times \mathcal{X}$.

Lemma 5 (Affine Representation 1) Suppose that absence of unanimity over outcomes holds. Then, $\succsim$ satisfies the acceptance principle and independence over identity lotteries with degenerate outcome lotteries if and only if there exist a continuous function $W: \triangle(\mathcal{I}) \times \mathcal{X} \rightarrow \mathbb{R}$ that represents $\succsim$ restricted to $\triangle(\mathcal{I}) \times \mathcal{X}$ and, for each individual $i$ in $\mathcal{I}$, a function $W_{i}: \triangle(\mathcal{X}) \rightarrow \mathbb{R}$ that represents $\succsim_{i}$ restricted to $\mathcal{X}$, such that for all $(z, x)$ in $\triangle(\mathcal{I}) \times \mathcal{X}$,

$$
W(z, x)=\sum_{i=1}^{I} z_{i} W_{i}(x) .
$$

Moreover, the functions $W_{i}$ are unique up to common affine transformations. 
The uniqueness of the functions $W_{i}$ follows because absence of unanimity implies the restriction of $\succsim$ to $\triangle(\mathcal{I}) \times \mathcal{X}$ satisfies the following property that we showed in the proof of Theorem 1 in Grant et al [7] guarantees uniqueness of the affine representation in (7).

Property U For each non-extreme (wrt $\succsim)$ identity lottery/outcome pair $(z, x)$ in $\triangle(\mathcal{I}) \times \mathcal{X}$, there exists an outcome $y$ in $\mathcal{X}$ and two individuals $i$ and $j$ in $\mathcal{I}$ such that $(i, y) \succ(z, x) \succ$ $(j, y)$.

To show that this property holds, consider any non-extreme (with respect to $\succsim)(z, x) \in$ $\Delta(\mathcal{I}) \times \mathcal{X}$. Let $(\hat{z}, \hat{x})$ be maximal with respect to $\succsim$ and $\left(z^{\prime}, x^{\prime}\right)$ be minimal with respect to $\succsim$ on $\Delta(\mathcal{I}) \times \mathcal{X}$. These maxima and minima exist because $\succsim$ is continuous and both $\Delta(\mathcal{I})$ and $\mathcal{X}$ are compact. Because $(\hat{z}, \hat{x})$ is maximal, by independence over lotteries with degenerate outcomes, there exists an $i \in \mathcal{I}$ such that $(i, \hat{x}) \sim(\hat{z}, \hat{x})$. Similarly, there exists a $j \in \mathcal{I}$ such that $\left(j, x^{\prime}\right) \sim\left(z^{\prime}, x^{\prime}\right)$. (It could be that $\hat{z}$ is the degenerate identity lottery $[i]$ or that $z^{\prime}$ is the degenerate identity lottery $[j]$.) Because $(z, x)$ is non-extreme, we therefore have $(i, \hat{x}) \succ(z, x) \succ\left(j, x^{\prime}\right)$. If $(i, \hat{x}) \sim\left(i, x^{\prime}\right)$, the result follows by setting $y=x^{\prime}$. Otherwise, $(i, \hat{x}) \succ\left(i, x^{\prime}\right)$ and, therefore, $\hat{x} \succ_{i} x^{\prime}$ by acceptance. By absence of unanimity over outcomes, there then exists a $k \in \mathcal{I}$ such that $x^{\prime} \succ_{k} \hat{x}$. By acceptance, $\left(k, x^{\prime}\right) \succ(k, \hat{x})$. If $(z, x) \succeq\left(k, x^{\prime}\right)$, then $(i, \hat{x}) \succ(z, x) \succ(k, \hat{x})$ and the proof is complete. Otherwise, we have $\left(k, x^{\prime}\right) \succ(z, x) \succ\left(j, x^{\prime}\right)$ and again the proof is complete. $^{11}$

\section{Step 2. Affine in outcome lotteries representation of $\succsim$.}

First we show, given redistributive scope, we need at most two identity lotteries $z^{1}$ and $z_{2}$, to 'cover' the entire range of the observer's preferences in the following sense: for all product lotteries $(z, \ell)$ either $(z, \ell) \sim\left(z^{1}, \ell^{\prime}\right)$ for some $\ell^{\prime}$, or $(z, \ell) \sim\left(z_{2}, \ell^{\prime \prime}\right)$ for some $\ell^{\prime \prime}$, or both. Moreover the set of product lotteries for which 'both' applies are not all indifferent.

To state this more formally, let the identity lotteries $z^{1}$ and $z_{2}$ (not necessarily distinct) and outcome lotteries $\ell^{1}, \ell_{2}$ (not necessarily distinct) be such that $\left(z^{1}, \ell^{1}\right) \succ\left(z_{2}, \ell_{2}\right)$ and such that $\left(z^{1}, \ell^{1}\right) \succsim(z, \ell) \succsim\left(z_{2}, \ell_{2}\right)$ for all product lotteries $(z, \ell)$. That is, the product lottery $\left(z^{1}, \ell^{1}\right)$ is

\footnotetext{
${ }^{11}$ We thank John Weymark for providing us with this compact and elegant proof.
} 
weakly better than all other product lotteries, and the product lottery $\left(z_{2}, \ell_{2}\right)$ is weakly worse than all other product lotteries. And let the outcome lotteries $\ell_{1}$ and $\ell^{2}$ (not necessarily distinct) be such that $\left(z^{1}, \ell^{1}\right) \succsim\left(z^{1}, \ell\right) \succsim\left(z^{1}, \ell_{1}\right)$ for all product lotteries $\left(z^{1}, \ell\right)$, and $\left(z_{2}, \ell^{2}\right) \succsim\left(z_{2}, \ell\right) \succsim\left(z_{2}, \ell_{2}\right)$ for all product lotteries $\left(z_{2}, \ell\right)$. That is, given the identity lottery $z^{1}$, the outcome lottery $\ell_{1}$ is (weakly) worse than all other outcome lotteries; and, given the identity lottery $z_{2}$, the outcome lottery $\ell^{2}$ is (weakly) better than all other outcome lotteries. The existence of these special lotteries follows from the continuity of $\succsim$, the non-emptiness of $\succ$, and the compactness of $\Delta(\mathcal{I}) \times \Delta(\mathcal{X})$. Moreover, by independence over outcome lotteries, we can take $\ell^{1}, \ell_{1}, \ell^{2}$, and $\ell_{2}$ each to be a degenerate outcome lottery. Let these be $x^{1}, x_{1}, x^{2}$, and $x_{2}$, respectively. This in turn means that by independence over identity lotteries with degenerate outcome lotteries, we can take $z^{1}$ and $z_{2}$ each to be a degenerate identity lottery. Let these be $i^{1}$ and $i_{2}$, respectively.

The following result follows from an analogous argument used in Grant et al [6] to prove their Lemma 7 (also called spanning). The roles of identities and outcomes are reversed and redistributive scope here plays the role that axiom of absence of unanimity played there.

Lemma 6 (Spanning) Assume redistributive scope applies and that the observer satisfies acceptance and independence over outcome lotteries. Let $i^{1}, i_{2}, x^{1}, x_{1}, x^{2}$, and $x_{2}$ be defined as above. Then, (a) either $\left(i^{1}, x_{1}\right) \sim\left(i_{2}, x_{2}\right)$ or $\left(i_{2}, x^{2}\right) \sim\left(i^{1}, x^{1}\right)$ or $\left(i_{2}, x^{2}\right) \succ\left(i^{1}, x_{1}\right)$ and (b) for all product lotteries $(z, \ell)$, either $\left(i^{1}, x^{1}\right) \succsim(z, \ell) \succsim\left(i^{1}, x_{1}\right)$ or $\left(i_{2}, x^{2}\right) \succsim(z, \ell) \succsim\left(i_{2}, x_{2}\right)$ or both.

Proof. (a) If $i^{1}=i_{2}$, then the first two cases both hold. Otherwise, suppose that the first two cases do not hold; that is, $\left(i^{1}, x_{1}\right) \succ\left(i_{2}, x_{2}\right)$ and $\left(i^{1}, x^{1}\right) \succ\left(i_{2}, x^{2}\right)$. By the definition of $x_{1}$, we know that $\left(i^{1}, x_{2}\right) \succsim\left(i^{1}, x_{1}\right)$, and hence $\left(i^{1}, x_{2}\right) \succ\left(i_{2}, x_{2}\right)$. Using redistributive scope and acceptance, there must exist another outcome $x \neq x_{2}$ such that $\left(i_{2}, x\right) \succ\left(i^{1}, x\right)$. Again by the definition of $x_{1}$, we know that $\left(i^{1}, x\right) \succsim\left(i^{1}, x_{1}\right)$, and hence $\left(i_{2}, x\right) \succ\left(i^{1}, x_{1}\right)$. By the definition of $x^{2}$, we know that $\left(i_{2}, x^{2}\right) \succsim\left(i_{2}, x\right)$, and hence $\left(i_{2}, x^{2}\right) \succ\left(i^{1}, x_{1}\right)$, as desired. Part (b) follows immediately from (a).

With Lemma 6 in hand, we can now construct the affine in outcome lottery representation of $\succsim$. The construction is analogous to one used in the proof of Lemma 8 in Grant et al [6] with 
the roles of identities and outcomes reversed and absence of unanimity replaced by redistributive scope.

Lemma 7 (Affine Representation 2) Suppose redistributive scope applies. Then, the observer satisfies the acceptance principle and independence over outcome lotteries if and only if there exist a continuous function $V: \triangle(\mathcal{I}) \times \Delta(\mathcal{X}) \rightarrow \mathbb{R}$ that represents $\succsim$ and is affine in its second argument.

Proof. Let $i^{1}, i_{2}, x^{1}, x_{1}, x^{2}$, and $x_{2}$ be defined as in Lemma 6 above. Given continuity, an immediate consequence of Lemma 6 is that, for all product lotteries $(z, \ell)$, either $(z, \ell) \sim\left(i^{1}, \ell^{\prime}\right)$ for some $\ell^{\prime}$, or $(z, \ell) \sim\left(i_{2}, \ell^{\prime \prime}\right)$ for some $\ell^{\prime \prime}$ or both. Moreover, we can choose the $\ell^{\prime}$ such that its support only contains outcomes $x^{1}$ and $x_{1}$. And similarly for $\ell^{\prime \prime}$ with respect to outcomes $x^{2}$ and $x_{2}$.

The proof of lemma now proceeds with two cases.

Case (1) The easiest case to consider is where $i^{1}=i_{2}$. In this case, $\left(i^{1}, x^{1}\right) \succ\left(i^{1}, x_{1}\right)$ and $\left(i^{1}, x^{1}\right) \succsim(z, \ell) \succsim\left(i^{1}, x_{1}\right)$, for all $(z, \ell)$. Then, for each $(z, \ell)$, let $V(z, \ell)$ be defined by

$$
\left(i^{1}, V(z, \ell)\left[x^{1}\right]+(1-V(z, \ell))\left[x_{1}\right]\right) \sim(z, \ell)
$$

By continuity and independence over outcome lotteries, such a $V(z, \ell)$ exists and is unique.

To show that this representation is affine, notice that if $\left(i^{1}, V(z, \ell)\left[x^{1}\right]+(1-V(z, \ell))\left[x_{1}\right]\right) \sim$ $(z, \ell)$ and $\left(i^{1}, V\left(z, \ell^{\prime}\right)\left[x^{1}\right]+\left(1-V\left(z, \ell^{\prime}\right)\right)\left[x_{1}\right]\right) \sim\left(z, \ell^{\prime}\right)$, then independence over outcome lotteries implies $\left(i^{1},\left[\alpha V(z, \ell)+(1-\alpha) V\left(z, \ell^{\prime}\right)\right]\left[x^{1}\right]+\left[1-\alpha V(z, \ell)-(1-\alpha) V\left(z, \ell^{\prime}\right)\right]\left[x_{1}\right]\right) \sim\left(z, \alpha \ell+(1-\alpha) \ell^{\prime}\right)$. Hence $\alpha V(z, \ell)+(1-\alpha) V\left(z, \ell^{\prime}\right)=V\left(z, \alpha \ell+(1-\alpha) \ell^{\prime}\right)$, as required.

Case (2). If $\left(i^{1}, x_{1}\right) \sim\left(i_{2}, x_{2}\right)$, then $\left(i^{1}, x^{1}\right) \succsim(z, \ell) \succsim\left(i^{1}, x_{1}\right)$ for all $(z, \ell)$ and hence case (1) applies. Similarly, if $\left(i_{2}, x^{2}\right) \sim\left(i^{1}, x^{1}\right)$, then $\left(i_{2}, x^{2}\right) \succsim(z, \ell) \succsim\left(i_{2}, x_{2}\right)$ for all $(z, \ell)$, and again case (1) applies (with $i_{2}$ in place of $\left.i^{1}\right)$. Hence suppose that $\left(i^{1}, x^{1}\right) \succ\left(i_{2}, x^{2}\right)$ and that $\left(i^{1}, x_{1}\right) \succ\left(i_{2}, x_{2}\right)$. Then, by Lemma $6,\left(i^{1}, x^{1}\right) \succ\left(i_{2}, x^{2}\right) \succ\left(i^{1}, x_{1}\right) \succ\left(i_{2}, x_{2}\right)$; that is, we have two overlapping intervals that 'span' the entire range of the observer's preferences.

Then, just as in case (1), we can construct an affine function $V^{1}(\cdot, \cdot)$ to represent the observer's preferences $\succsim$ restricted to those $(z, \ell)$ such that $\left(i^{1}, x^{1}\right) \succsim(z, \ell) \succsim\left(i^{1}, x_{1}\right)$, and we can construct 
an affine function $V^{2}(\cdot, \cdot)$ to represent $\succsim$ restricted to those $(z, \ell)$ such that $\left(i_{2}, x^{2}\right) \succsim(z, \ell) \succsim$ $\left(i_{2}, x_{2}\right)$. We can then apply an affine re-normalization of either $V^{1}$ or $V^{2}$ such the (re-normalized) representations agree on the 'overlap' $\left(i_{2}, x^{2}\right) \succsim(z, \ell) \succsim\left(i^{1}, x_{1}\right)$. Since $V^{1}(\cdot, \cdot)$ and $V^{2}(\cdot, \cdot)$ are affine, the re-normalized representation is affine.

\section{Step 3. Combining the representations obtained in Steps 1 and 2.}

In summary, we have shown from Step 1 that $W(z, x)=\sum_{i} z_{i} W_{i}(x)$ represents $\succsim$ restricted to $\triangle(\mathcal{I}) \times \mathcal{X}$ and from Step 2 that $V(z, \ell)$ is a representation of $\succsim$ that is affine in $\ell$, that is, $V(z, \ell)=\int V(z, x) \ell(d x)$.

For $i=1, \ldots, I$, set $U_{i}(\ell):=V([i], \ell)$. By acceptance, it follows that $U_{i}(\ell)$ is a(n expected utility) representation of $\succsim_{i}$.

Notice that $W_{i}(x)$ is a monotonic transformation of $U_{i}(x)$. Let $\varphi:\left[V\left(i_{2}, x_{2}\right), V\left(i^{1}, x^{1}\right)\right] \rightarrow$ $\left[W_{i_{2}}\left(x_{2}\right), W_{i^{1}}\left(x^{1}\right)\right]$ be the monotonic transformation implicitly defined by $W_{i}(x)=\varphi \circ U_{i}(x) .{ }^{12}$ Also notice that $V(z, x)$ is a monotonic transformation of $W(z, x)$. Let $\xi:\left[W\left(i_{2}, x_{2}\right), W\left(i^{1}, x^{1}\right)\right] \rightarrow$ $\left[V\left(i_{2}, x_{2}\right), V\left(i^{1}, x^{1}\right)\right]$, implicitly be defined by $V(z, x)=\xi \circ W(z, x)$.

By construction we have, for any $i \in \mathcal{I}$ and any $x \in \mathcal{X}, V(i, x)=\xi \circ W_{i}(x)=\xi \circ \varphi \circ U_{i}(x)$. Hence $\xi=\varphi^{-1}$. Putting this all together we obtain,

$$
\begin{aligned}
V(z, \ell) & =\int \varphi^{-1} \circ W(z, x) \ell(d x)=\int \varphi^{-1}\left(\sum_{i} z_{i} W_{i}(x)\right) \ell(d x) \\
& =\int \varphi^{-1}\left(\sum_{i} z_{i} \varphi \circ U_{i}(x)\right) \ell(d x)
\end{aligned}
$$

as desired.

\section{Proof of Proposition 3 (Concavity)}

\section{Concavity $\Rightarrow$ Preference for Life-Chances.}

Suppose $\left(z, \ell^{\prime}\right) \sim\left(z^{\prime}, \ell\right)$. Using the fact that the representation is affine in its second argument, we have $V\left(z, \alpha \ell+(1-\alpha) \ell^{\prime}\right)=\alpha V(z, \ell)+(1-\alpha) V\left(z, \ell^{\prime}\right)$. Using the fact that $\left(z, \ell^{\prime}\right) \sim\left(z^{\prime}, \ell\right)$, the last expression is equal to $\alpha V(z, \ell)+(1-\alpha) V\left(z^{\prime}, \ell\right)$. But the concavity $\varphi$ implies that $\varphi^{-1}$

\footnotetext{
12 For a given $i, U_{i}(\mathcal{X})$ may only be a subset of the domain of $\varphi$. However, by Lemma 6 , it is clear that a single $\varphi$ can be used for the entire domain.
} 
is convex, and so,

$$
\begin{aligned}
& \alpha V(z, \ell)+(1-\alpha) V\left(z^{\prime}, \ell\right) \\
= & \int\left[\alpha \varphi^{-1} \circ\left(\sum_{i} z_{i} \varphi\left(U_{i}(x)\right)\right)+(1-\alpha) \varphi^{-1} \circ\left(\sum_{i} z_{i}^{\prime} \varphi\left(U_{i}(x)\right)\right)\right] \ell(d x) \\
\geq & \int \varphi^{-1} \circ\left(\sum_{i}\left[\alpha z_{i}+(1-\alpha) z_{i}^{\prime}\right] \varphi\left(U_{i}(x)\right)\right) \ell(d x) \\
= & V\left(\alpha z+(1-\alpha) z^{\prime}, \ell\right) .
\end{aligned}
$$

Hence, the observer exhibits a preference for life chances.

\section{Preference for Life-Chances $\Rightarrow$ Concavity}

If $\varphi^{-1}$ is not convex, then there exist $v<w$ in the domain of $\varphi^{-1}$ (i.e., the set

$$
\left\{\sum_{i} z_{i} \varphi\left(U_{i}(x)\right): z \in \triangle(\mathcal{I}), x \in \mathcal{X}\right\}
$$

which by the richness conditions is an interval of the form $\left.\left[\varphi\left(U_{i_{2}}\left(x_{2}\right)\right), \varphi\left(U_{i^{1}}\left(x^{1}\right)\right)\right]\right)$ such that

$$
\alpha \varphi^{-1}(v)+(1-\alpha) \varphi^{-1}(w)<\varphi^{-1}(\alpha v+(1-\alpha) w)
$$

holds for some $\alpha$ in $(0,1)$. Note that the continuity of $\varphi$ implies that the set of pairs $(v, w)$ satisfying this inequality is an open set. Moreover, there exists $u \in(v, w)$ and $\varepsilon>0$ such that the inequality is satisfied for all $v^{\prime} \in(u-\varepsilon, u)$ and $w^{\prime} \in(u, u+\varepsilon) .{ }^{13}$ Fix such $v^{\prime}$ and $w^{\prime}$. By definition, there exists an identity lottery $z$ and an outcome $x$ such that $\sum_{i} z_{i} \varphi\left(U_{i}(x)\right)=v^{\prime}$ (or, equivalently, $\left.V(z, x)=\varphi^{-1}\left(v^{\prime}\right)\right)$. Without loss of generality, all $z_{i}>0$ and not all $U_{i}(x)$ are the same. ${ }^{14}$ Hence, for $\varepsilon$ sufficiently small there exists an identity lottery $z^{\prime}$ satisfying $\sum_{i} z_{i}^{\prime} \varphi\left(U_{i}(x)\right)=w^{\prime}$ (and $\left.V\left(z^{\prime}, x\right)=\varphi^{-1}\left(w^{\prime}\right)\right)$. Choose $x^{\prime \prime}$ such that $\sum_{i} z_{i} \varphi\left(U_{i}\left(x^{\prime \prime}\right)\right)$ is equal to some $v^{\prime \prime} \neq v^{\prime}$ (if, for all $x^{\prime \prime}, \sum_{i} z_{i} \varphi\left(U_{i}\left(x^{\prime \prime}\right)\right)=v^{\prime}$, then, as not all individuals' preferences agree on the order of the outcomes, a slight change in $z$ would allow it). Without loss of generality $v^{\prime \prime}>v^{\prime}$. Consider

${ }^{13}$ Let $a^{*}$ be the maximal $a$ for which the intersection of the line $a+b t, b=\frac{\varphi^{-1}(w)-\varphi^{-1}(v)}{w-v}$, and the graph of $\varphi^{-1}$ over $(v, w)$ is non empty. Then, $u$ is the minimal $t$ such that $\left(t, \varphi^{-1}(t)\right)$ is a tangency point of $a^{*}+b t$

${ }^{14}$ If some $z_{i}=0$, then $v^{\prime}$ can be slightly changed. If $U_{i}(x)=U_{j}(x)$ for all $i, j$, then $\sum_{i} z_{i}^{\prime} \varphi\left(U_{i}(x)\right)$ is independent of $z^{\prime}$ and, by absence of unanimity, a slight change in $v^{\prime}$ gives $x^{\prime}$ for which $U_{i}\left(x^{\prime}\right) \neq U_{j}\left(x^{\prime}\right)$ for some $i, j$ (otherwise there is a unanimus preference of $x$ over $x^{\prime}$, or vice versa) 
$\ell=(1-\delta)[x]+\delta\left[x^{\prime \prime}\right]$. Then,

$$
\begin{aligned}
V(z, \ell) & =(1-\delta) \varphi^{-1}\left(\sum_{i} z_{i} \varphi\left(U_{i}(x)\right)\right)+\delta \varphi^{-1}\left(\sum_{i} z_{i} \varphi\left(U_{i}\left(x^{\prime \prime}\right)\right)\right) \\
& =(1-\delta) \varphi^{-1}\left(v^{\prime}\right)+\delta \varphi^{-1}\left(v^{\prime \prime}\right) .
\end{aligned}
$$

Hence, if $\varepsilon$ is chosen sufficiently small so that $w^{\prime}<v^{\prime \prime}$, then there exists $\delta^{\prime}$ such that $\ell^{\prime}=$ $\left(1-\delta^{\prime}\right)[x]+\delta^{\prime}\left[x^{\prime \prime}\right]$ satisfies $V\left(z, \ell^{\prime}\right)=\varphi^{-1}\left(w^{\prime}\right)$. Summarizing,

$$
V\left(z^{\prime}, x\right)=\varphi^{-1}\left(w^{\prime}\right)=V\left(z, \ell^{\prime}\right) \text { and }\left(z^{\prime}, x\right) \sim\left(z, \ell^{\prime}\right)
$$

But then we would have (for some $\alpha$ in $(0,1)$ ),

$$
\begin{aligned}
& V\left(z, \alpha[x]+(1-\alpha) \ell^{\prime}\right)=\alpha V(z, x)+(1-\alpha) V\left(z, \ell^{\prime}\right) \\
= & \alpha \varphi^{-1}\left(v^{\prime}\right)+(1-\alpha) \varphi^{-1}\left(w^{\prime}\right)<\varphi^{-1}\left(\alpha v^{\prime}+(1-\alpha) w^{\prime}\right) \\
= & \varphi^{-1}\left(\sum_{i}\left[\alpha z_{i}+(1-\alpha) z_{i}^{\prime}\right] \varphi\left(U_{i}(x)\right)\right)=V\left(\alpha z+(1-\alpha) z^{\prime}, x\right),
\end{aligned}
$$

a violation of preference for life chances.

Proof of Theorem 4 (Utilitarianism). It is clear that (b) implies (a). We will show (a) $\Rightarrow(b)$. We observed in Grant et al [6, p. 1955] that independence over outcome lottteries implies a property we called indifference between individuals facing similar risks. It thus follows from Theorem 1 and Proposition 6 in Grant et al [6, p. 1947 and p. 1952, respectively] that the preferences may be represented by a generalized utilitarian representation with common utility transformation function, that is, $V(z, \ell)=\sum_{i=1}^{I} z_{i} \phi\left[\hat{U}_{i}(\ell)\right]$. It is enough to show that this common $\phi$-function is affine. Since the proof is long, we will break it into 6 steps, and we will signpost some parts.

Step 1 is to prove the following lemma that shows the function $\phi^{-1} \circ V$ is affine on $\triangle(\mathcal{X})$, from which it immediately follows that

$$
\begin{aligned}
\hat{V}(z, \ell) & =\int_{\mathcal{X}} \phi^{-1} \circ V(z,[x]) \ell(d x) \\
& =\int_{\mathcal{X}} \phi^{-1}\left(\sum_{i=1}^{n} z_{i} \phi\left(\hat{U}_{i}(x)\right)\right) \ell(d x)
\end{aligned}
$$

is an expected equally-distributed equivalent-utility representation of the observer's preferences. 
Lemma 8 Suppose that absence of unanimity and redistributive scope both apply, and that the observer satisfies the acceptance principle, independence over outcome lotteries and independence over identity lotteries. Let $V$ and $\phi$ be defined so that, $V(z, \ell)=\sum_{i=1}^{I} z_{i} \phi\left[\hat{U}_{i}(\ell)\right]$ and V represents the observer's preferences. Then, for each $z$ in $\triangle(\mathcal{I})$, the function $\phi^{-1} \circ V(z, \cdot): \triangle(\mathcal{X}) \rightarrow \mathbb{R}$ is affine.

Proof. Fix an identity lottery $z$ and an individual $i$. Let $\widehat{\mathcal{U}}_{i} \subset \mathbb{R}$ be the interval such that $u \in \widehat{\mathcal{U}}_{i}$ implies that there exists an $\ell$ such that $\hat{U}_{i}(\ell)=u$. We will first show that $\phi^{-1} \circ V(z, \cdot)$ is affine on the inverse image of $\widehat{\mathcal{U}}_{i}$; that is, on the subset of outcome lotteries $\{\ell \in \triangle(\mathcal{X})$ : $\left.\phi^{-1} \circ V(z, \ell) \in \widehat{\mathcal{U}}_{i}\right\}$. If this inverse image is empty, then affinity is trivial. Hence, consider two outcome lotteries $\ell, \ell^{\prime}$ (not necessarily distinct) such that $\phi^{-1} \circ V(z, \ell) \in \widehat{\mathcal{U}}_{i}$ and $\phi^{-1} \circ V\left(z, \ell^{\prime}\right) \in \widehat{\mathcal{U}}_{i}$. By the definition of $\widehat{\mathcal{U}}_{i}$, there exist two outcome lotteries $\bar{\ell}$ and $\bar{\ell}^{\prime}$ such that $\phi^{-1} \circ V(z, \ell)=\hat{U}_{i}(\bar{\ell})$ and $\phi^{-1} \circ V\left(z, \ell^{\prime}\right)=\hat{U}_{i}\left(\bar{\ell}^{\prime}\right)$; that is, $(z, \ell) \sim(i, \bar{\ell})$ and $\left(z, \ell^{\prime}\right) \sim\left(i, \bar{\ell}^{\prime}\right)$. Applying independence over outcome lotteries yields

$$
\left(z, \alpha \ell+(1-\alpha) \ell^{\prime}\right) \sim\left(i, \alpha \bar{\ell}+(1-\alpha) \bar{\ell}^{\prime}\right)
$$

for all $\alpha$ in $[0,1]$. Hence, $\phi^{-1} \circ V\left(z, \alpha \ell+(1-\alpha) \ell^{\prime}\right) \in \widehat{\mathcal{U}}_{i}$. Applying the representation yields:

$$
\begin{aligned}
\phi^{-1} \circ V\left(z, \alpha \ell+(1-\alpha) \ell^{\prime}\right) & =\hat{U}_{i}\left(\alpha \bar{\ell}+(1-\alpha) \bar{\ell}^{\prime}\right) \\
& \left.=\alpha \hat{U}_{i}(\bar{\ell})+(1-\alpha) \hat{U}_{i}\left(\bar{\ell}^{\prime}\right) \quad \text { (by affinity of } \hat{U}_{i}\right) \\
& =\alpha \phi^{-1} \circ V(z, \ell)+(1-\alpha) \phi^{-1} \circ V\left(z, \ell^{\prime}\right) .
\end{aligned}
$$

where the third equality is by the definition of $\bar{\ell}$ and $\bar{\ell}^{\prime}$. This argument holds for all $i$.

An immediate consequence of Lemma 6 is that there exist two individuals $i^{1}$ and $i_{2}$ such that range $\left[\phi^{-1} \circ V(z, \cdot)\right] \subseteq \widehat{\mathcal{U}}_{i^{1}} \cup \widehat{\mathcal{U}}_{i_{2}}$ and the inverse image of $\widehat{\mathcal{U}}_{i^{1}} \cup \widehat{\mathcal{U}}_{i_{2}}$ is $\Delta(\mathcal{X})$. We know that $\phi^{-1} \circ V(z, \cdot)$ is affine on the inverse image of $\widehat{\mathcal{U}}_{i^{1}}$ and $\widehat{\mathcal{U}}_{i_{2}}$. Moreover, by Lemma 6 , the interior of $\widehat{\mathcal{U}}_{i^{1} i_{2}}\left(=\right.$ interior $\left.\widehat{\mathcal{U}}_{i^{1}} \cap \widehat{\mathcal{U}}_{i_{2}}\right)$ is not empty. Hence $\phi^{-1} \circ V(z, \cdot)$ is affine on $\Delta(\mathcal{X})$. This argument holds for all $z$.

It follows from Lemma 7 in Grant et al [6, p. 1960] that absence of unanimity, acceptance and independence over identity lotteries imply there exist individuals $i^{1}, i_{1}, i^{2}$ and $i_{2}$ (not necessarily 
distinct) and outcome lotteries $\ell^{1}$ and $\ell_{2}$ (also not necessarily distinct) such that (i) $\left(i^{1}, \ell^{1}\right) \succ$ $\left(i_{2}, \ell_{2}\right)$ and such that $\left(i^{1}, \ell^{1}\right) \succsim(z, \ell) \succsim\left(i_{2}, \ell_{2}\right)$, for any product lottery $(z, \ell)$, (ii) either $\left(i_{1}, \ell^{1}\right) \sim$ $\left(i_{2}, \ell_{2}\right)$ or $\left(i^{2}, \ell_{2}\right) \sim\left(i^{1}, \ell^{1}\right)$ or $\left(i^{2}, \ell_{2}\right) \succ\left(i_{1}, \ell^{1}\right)$, and (iii) for any product lottery $(z, \ell)$ either $\left(i^{1}, \ell^{1}\right) \succsim(z, \ell) \succsim\left(i_{1}, \ell^{1}\right)$ or $\left(i^{2}, \ell_{2}\right) \succsim(z, \ell) \succsim\left(i_{2}, \ell_{2}\right)$ or both. Now since we also have independence over outcome lotteries we can take each $\ell^{1}$ and $\ell_{2}$ to be a degenerate outcome lottery. Let these be $x^{1}$ and $x_{2}$.

Recall that, given our representation with a common $\phi$-function, $(i, \ell) \sim\left(j, \ell^{\prime}\right)$ implies $\hat{U}_{i}(\ell)=$ $\hat{U}_{j}\left(\ell^{\prime}\right)$. Hence, from (ii) either $\hat{U}_{i_{1}}\left(x^{1}\right)=\hat{U}_{i_{2}}\left(x_{2}\right)$ or $\hat{U}_{i^{2}}\left(x_{2}\right)=\hat{U}_{i^{1}}\left(x^{1}\right)$ or $\hat{U}_{i^{2}}\left(x_{2}\right)>\hat{U}_{i_{1}}\left(x^{1}\right)$. Notice that for all product lotteries $(z, \ell)$, we have $\phi^{-1} \circ V(z, \ell)=\phi^{-1}\left(\sum_{i} z_{i} \phi\left[\hat{U}_{i}(\ell)\right]\right) \in$ $\left[\hat{U}_{i_{1}}\left(x^{1}\right), \hat{U}_{i^{1}}\left(x^{1}\right)\right] \cup\left[\hat{U}_{i_{2}}\left(x_{2}\right), \hat{U}_{i^{2}}\left(x_{2}\right)\right]$. We will first concentrate on the interval $\left[\hat{U}_{i_{1}}\left(x^{1}\right), \hat{U}_{i^{1}}\left(x^{1}\right)\right]$, but we will return to the interval $\left[\hat{U}_{i_{2}}\left(x_{2}\right), \hat{U}_{i^{2}}\left(x_{2}\right)\right]$ in Step 5. If $\hat{U}_{i_{1}}\left(x^{1}\right)=\hat{U}_{i^{1}}\left(x^{1}\right)$, then affinity of $\phi^{-1} \circ V(z, \cdot)$ on the interval $\left[\hat{U}_{i_{1}}\left(x^{1}\right), \hat{U}_{i^{1}}\left(x^{1}\right)\right]$ is trivial. ${ }^{15}$ Hence, assume $\hat{U}_{i_{1}}\left(x^{1}\right)<$ $\hat{U}_{i^{1}}\left(x^{1}\right)$.

Defining $\hat{x}$ and $\bar{u}$. Since $\left(i^{1}, x^{1}\right) \succ\left(i_{1}, x^{1}\right)$, by redistributive scope, there exists an outcome $\hat{x}$ such that $\left(i_{1}, \hat{x}\right) \succ\left(i^{1}, \hat{x}\right)$. Consider the outcome lotteries $\ell_{[\lambda]}$ defined by $\ell_{[\lambda]}:=\lambda[\hat{x}]+(1-\lambda)\left[x^{1}\right]$. By the continuity of both $\hat{U}_{i_{1}}$ and $\hat{U}_{i^{1}}$, there must exist an outcome lottery $\bar{\ell}\left(:=\ell_{[\bar{\lambda}]}\right)$ such that $\left(i^{1}, \bar{\ell}\right) \sim\left(i_{1}, \bar{\ell}\right)$. Let $\bar{u}$ be given by

$$
\bar{u}:=\phi^{-1}\left[V\left(i^{1}, \bar{\ell}\right)\right]=\phi^{-1}\left[V\left(i_{1}, \bar{\ell}\right)\right]
$$

The level of utility $\bar{u}$ is going to be important in the argument below. By the definition of $x^{1}$, if $\bar{u}$ does not lie in the interval $\left[\hat{U}_{i_{1}}\left(x^{1}\right), \hat{U}_{i^{1}}\left(x^{1}\right)\right]$, then $\bar{u}<\hat{U}_{i_{1}}\left(x^{1}\right)$.

Step 2 is to show that, for all $u^{\prime}$ and $u^{\prime \prime} \in\left[\hat{U}_{i_{1}}\left(x^{1}\right), \hat{U}_{i^{1}}\left(x^{1}\right)\right]$ and all $\alpha$ and $\beta$ in $[0,1]$,

$$
\begin{aligned}
& \phi^{-1}\left[\beta \phi\left[\alpha u^{\prime}+(1-\alpha) \bar{u}\right]+(1-\beta) \phi\left[\alpha u^{\prime \prime}+(1-\alpha) \bar{u}\right]\right] \\
= & \alpha \phi^{-1}\left[\beta \phi\left(u^{\prime}\right)+(1-\beta) \phi\left(u^{\prime \prime}\right)\right]+(1-\alpha) \bar{u} .
\end{aligned}
$$

To show this, fix $u^{\prime}$ and $u^{\prime \prime} \in\left[\hat{U}_{i_{1}}\left(x^{1}\right), \hat{U}_{i^{1}}\left(x^{1}\right)\right]$. Denote by $z^{\prime}=\beta^{\prime}\left[i^{1}\right]+\left(1-\beta^{\prime}\right)\left[i_{1}\right]$ and $z^{\prime \prime}=\beta^{\prime \prime}\left[i^{1}\right]+\left(1-\beta^{\prime \prime}\right)\left[i_{1}\right]$, the identity lotteries with support just on $i^{1}$ and $i_{1}$ for which

${ }^{15}$ In this case, $\hat{U}_{i^{2}}\left(x_{2}\right)=\hat{U}_{i^{1}}\left(x^{1}\right)$ and hence showing that $\phi$ is affine on $\left[\hat{U}_{i_{2}}\left(x_{2}\right), \hat{U}_{i^{2}}\left(x_{2}\right)\right]$ would be enough. 
$\phi^{-1}\left[V\left(z^{\prime}, x^{1}\right)\right]=u^{\prime}$ and $\phi^{-1}\left[V\left(z^{\prime \prime}, x^{1}\right)\right]=u^{\prime \prime}$. Also fix $\alpha$ and $\beta$ in $[0,1]$, and define $u^{\beta}$ by:

$$
u^{\beta}:=\phi^{-1}\left[V\left(\beta z^{\prime}+(1-\beta) z^{\prime \prime}, x^{1}\right)\right] .
$$

By the definition of $\bar{u}$ and the fact that $V$ is affine in identity lotteries, we have

$$
\bar{u}=\phi^{-1}\left[V\left(\beta z^{\prime}+(1-\beta) z^{\prime \prime}, \bar{\ell}\right)\right] .
$$

By lemma 8 , the function $\phi^{-1} \circ V\left(\beta z^{\prime}+(1-\beta) z^{\prime \prime}, \cdot\right)$ is affine on $\Delta(\mathcal{X})$, hence combining expressions (10) and (11), we get

$$
\phi^{-1}\left[V\left(\beta z^{\prime}+(1-\beta) z^{\prime \prime}, \alpha\left[x^{1}\right]+(1-\alpha) \bar{\ell}\right)\right]=\alpha u^{\beta}+(1-\alpha) \bar{u}
$$

Our two affinity properties allow us to expand the left side of this expression . First, by the affinity of $V\left(\cdot, \alpha\left[x^{1}\right]+(1-\alpha) \bar{\ell}\right)$ on $\Delta(\mathcal{I})$, we get

$$
\begin{aligned}
& V\left(\beta z^{\prime}+(1-\beta) z^{\prime \prime}, \alpha\left[x^{1}\right]+(1-\alpha) \bar{\ell}\right) \\
= & \beta V\left(z^{\prime}, \alpha\left[x^{1}\right]+(1-\alpha) \bar{\ell}\right)+(1-\beta) V\left(z^{\prime \prime}, \alpha\left[x^{1}\right]+(1-\alpha) \bar{\ell}\right) \\
= & \beta \phi\left[\phi^{-1} \circ V\left(z^{\prime}, \alpha\left[x^{1}\right]+(1-\alpha) \bar{\ell}\right)\right]+(1-\beta) \phi\left[\phi^{-1} \circ V\left(z^{\prime \prime}, \alpha\left[x^{1}\right]+(1-\alpha) \bar{\ell}\right)\right]
\end{aligned}
$$

Second, by the affinity of $\phi^{-1} \circ V\left(z^{\prime}, \cdot\right)$ and $\phi^{-1} \circ V\left(z^{\prime \prime}, \cdot\right)$ on $\Delta(\mathcal{X})$, we have

$$
\begin{aligned}
{\left[\phi^{-1} \circ V\left(z^{\prime}, \alpha\left[x^{1}\right]+(1-\alpha) \bar{\ell}\right)\right]=} & {\left[\alpha \phi^{-1} \circ V\left(z^{\prime}, x^{1}\right)+(1-\alpha) \phi^{-1} \circ V\left(z^{\prime}, \bar{\ell}\right)\right] } \\
& \text { and } \\
{\left[\phi^{-1} \circ V\left(z^{\prime \prime}, \alpha\left[x^{1}\right]+(1-\alpha) \bar{\ell}\right)\right]=} & {\left[\alpha \phi^{-1} \circ V\left(z^{\prime \prime}, x^{1}\right)+(1-\alpha) \phi^{-1} \circ V\left(z^{\prime \prime}, \bar{\ell}\right)\right] . }
\end{aligned}
$$

Substituting $u^{\prime}=\phi^{-1} \circ V\left(z^{\prime}, x^{1}\right), u^{\prime \prime}=\phi^{-1} \circ V\left(z^{\prime \prime}, x^{1}\right)$ and $\bar{u}=\phi^{-1} \circ V\left(z^{\prime}, \bar{\ell}\right)=\phi^{-1} \circ V\left(z^{\prime \prime}, \bar{\ell}\right)$, expressions (14) and (15) become

$$
\left[\alpha u^{\prime}+(1-\alpha) \bar{u}\right] \quad \text { and } \quad\left[\alpha u^{\prime \prime}+(1-\alpha) \bar{u}\right]
$$

respectively. Substituting these back into expression (13) and then substituting back into the left side of expression (12) yields

$$
\phi^{-1}\left[\beta \phi\left[\alpha u^{\prime}+(1-\alpha) \bar{u}\right]+(1-\beta) \phi\left[\alpha u^{\prime \prime}+(1-\alpha) \bar{u}\right]\right]=\alpha u^{\beta}+(1-\alpha) \bar{u}
$$


Using the definition of $u^{\beta}$ in expression (10) and the affinity of $V\left(\cdot, x^{1}\right)$ on $\Delta(\mathcal{I})$, we have

$$
\begin{aligned}
u^{\beta} & =\phi^{-1}\left[V\left(\beta z^{\prime}+(1-\beta) z^{\prime \prime}, x^{1}\right)\right] \\
& =\phi^{-1}\left[\beta V\left(z^{\prime}, x^{1}\right)+(1-\beta) V\left(z^{\prime \prime}, x^{1}\right)\right] \\
& =\phi^{-1}\left[\beta \phi\left(\phi^{-1}\left[V\left(z^{\prime}, x^{1}\right)\right]\right)+(1-\beta) \phi\left(\phi^{-1}\left[V\left(z^{\prime \prime}, x^{1}\right)\right]\right)\right] \\
& =\phi^{-1}\left[\beta \phi\left(u^{\prime}\right)+(1-\beta) \phi\left(u^{\prime \prime}\right)\right]
\end{aligned}
$$

where the last line follows from the definitions of $u^{\prime}$ and $u^{\prime \prime}$. Substituting expression (17) back into expression (16) yields expression (9), as desired. Our choice of $u^{\prime}, u^{\prime \prime}, \alpha$ and $\beta$ was arbitrary, so this completes step 2.

Re-normalization. Recall that functions $\left[\hat{U}_{i}\right]_{i \in \mathcal{I}}$ are unique only up to a common affine transformation and that the composite functions $\left[\phi \circ \hat{U}_{i}\right]_{i \in \mathcal{I}}$ are also unique only up to a common affine transformation. Hence, we can re-normalize such that the utility level $\bar{u}=0$. With slight abuse of notation, we will continue to use $\phi$ and $\left[\hat{U}_{i}\right]_{i \in \mathcal{I}}$ to denote these re-normalized functions. With this re-normalization, expression (9) becomes

$$
\phi^{-1}\left[\beta \phi\left(\alpha u^{\prime}\right)+(1-\beta) \phi\left(\alpha u^{\prime \prime}\right)\right]=\alpha \phi^{-1}\left[\beta \phi\left(u^{\prime}\right)+(1-\beta) \phi\left(u^{\prime \prime}\right)\right] .
$$

Since $u^{\prime}$ and $u^{\prime \prime}$ were arbitrary, expression (18) holds (for all $\alpha$ and $\beta$ in $[0,1]$ ) for all utility pairs in the (re-normalized) interval $\left[\hat{U}_{i_{1}}\left(x^{1}\right), \hat{U}_{i^{1}}\left(x^{1}\right)\right]$. Recall that 0 need not lie in this interval.

Step 3 is to show that expression (18) also holds (for all $\alpha$ and $\beta$ in $[0,1]$ ) for all utility pairs $u^{\prime}$ and $u^{\prime \prime}$ in $\left[0, \hat{U}_{i^{1}}\left(x^{1}\right)\right]$ even if $0<\hat{U}_{i_{1}}\left(x^{1}\right)$; that is, even if $\bar{u}$ does not lie in $\left[\hat{U}_{i_{1}}\left(x^{1}\right), \hat{U}_{i^{1}}\left(x^{1}\right)\right]$. To show this, we will establish that expression (18) holds in each interval in a sequence of intervals $I_{0}, I_{1}, \ldots$, with (i) $I_{0}:=\left[\hat{U}_{i_{1}}\left(x^{1}\right), \hat{U}_{i^{1}}\left(x^{1}\right)\right]$; (ii) $I_{n} \cap I_{n+1}$ an interval with positive length (and so having a non-empty interior), for all $n=0,1, \ldots$; and (iii) $\bigcup_{n=0}^{\infty} I_{n}=\left(0, \hat{U}_{i^{1}}\left(x^{1}\right)\right]$. The continuity of $\phi(\cdot)$ then implies that (18) holds on all of $\left[0, \hat{U}_{i^{1}}\left(x^{1}\right)\right]$.

Fix an $\tilde{\alpha} \in(0,1)$ for which $\tilde{\alpha} \hat{U}_{i^{1}}\left(x^{1}\right)>\hat{U}_{i_{1}}\left(x^{1}\right)\left(>\tilde{\alpha} \hat{U}_{i_{1}}\left(x^{1}\right)\right)$. Set $I_{n}:=\left[\tilde{\alpha}^{n} \hat{U}_{i_{1}}\left(x^{1}\right), \tilde{\alpha}^{n} \hat{U}_{i^{1}}\left(x^{1}\right)\right]$. By construction, $I_{n} \cap I_{n+1}$ is an interval with positive length and $\bigcup_{n=0}^{\infty} I_{n}=\left(0, \hat{U}_{i^{1}}\left(x^{1}\right)\right]$. To see that $I_{n}$ satisfies (18), consider a pair of utilities $u^{\prime}$ and $u^{\prime \prime}$ in $I_{n}$ and fix $\alpha, \beta$ in $[0,1]$. By 
construction, both $\hat{u}^{\prime}:=u^{\prime} / \tilde{\alpha}^{n}$ and $\hat{u}^{\prime \prime}:=u^{\prime \prime} / \tilde{\alpha}^{n}$ are in $\left[\hat{U}_{i_{1}}\left(x^{1}\right), \hat{U}_{i^{1}}\left(x^{1}\right)\right]$. Since $\tilde{\alpha}^{n}$ and $\alpha \tilde{\alpha}^{n}$ are in $[0,1]$, expression (18) implies

$$
\phi^{-1}\left[\beta \phi\left(\tilde{\alpha}^{n} \hat{u}^{\prime}\right)+(1-\beta) \phi\left(\tilde{\alpha}^{n} \hat{u}^{\prime \prime}\right)\right]=\tilde{\alpha}^{n} \phi^{-1}\left[\beta \phi\left(\hat{u}^{\prime}\right)+(1-\beta) \phi\left(\hat{u}^{\prime \prime}\right)\right]
$$

and

$$
\phi^{-1}\left[\beta \phi\left(\alpha \tilde{\alpha}^{n} \hat{u}^{\prime}\right)+(1-\beta) \phi\left(\alpha \tilde{\alpha}^{n} \hat{u}^{\prime \prime}\right)\right]=\alpha \tilde{\alpha}^{n} \phi^{-1}\left[\beta \phi\left(\hat{u}^{\prime}\right)+(1-\beta) \phi\left(\hat{u}^{\prime \prime}\right)\right] .
$$

Substituting $u^{\prime}$ for $\tilde{\alpha}^{n} \hat{u}^{\prime}$ and $u^{\prime \prime}$ for $\tilde{\alpha}^{n} \hat{u}^{\prime \prime}$ and then combining expressions (19) and (20), we obtain

$$
\phi^{-1}\left[\beta \phi\left(\alpha u^{\prime}\right)+(1-\beta) \phi\left(\alpha u^{\prime \prime}\right)\right]=\alpha \phi^{-1}\left[\beta \phi\left(u^{\prime}\right)+(1-\beta) \phi\left(u^{\prime \prime}\right)\right],
$$

as required.

Step 4 consists of the following lemma showing that $\phi$ must be an affine transformation of a homogenous function.

Lemma 9 Suppose that $\phi(\cdot)$ satisfies equation (18) for all $u^{\prime}, u^{\prime \prime}$ in $\left[\min \left\{0, \hat{U}_{i_{1}}\left(x^{1}\right)\right\}, \hat{U}_{i^{1}}\left(x^{1}\right)\right]$ and all $\alpha$ and $\beta$ in $[0,1]$, then

$$
\phi(u)=\left\{\begin{array}{cc}
C u^{k}+D & u \geq 0 \\
-C(-u)^{k}+D & u<0
\end{array}\right.
$$

for some $C, k$ in $\mathbb{R}_{++}$and some $D$ in $\mathbb{R}$.

Case $1 .{ }^{16} \quad \hat{U}_{i_{1}}\left(x^{1}\right) \geq 0$. We shall show that

$$
\phi\left(\alpha u^{\prime \prime}\right)-\phi\left(\alpha u^{\prime}\right)=\gamma(\alpha)\left[\phi\left(u^{\prime \prime}\right)-\phi\left(u^{\prime}\right)\right]
$$

for all $u^{\prime}, u^{\prime \prime} \in\left[0, \hat{U}_{i^{1}}\left(x^{1}\right)\right]$ and all $\alpha \in(0,1)$.

Consider four positive numbers $u_{1}, \hat{u}_{1}, u_{2}, \hat{u}_{2}$ in $\left[\hat{U}_{i_{1}}\left(x^{1}\right), \hat{U}_{i^{1}}\left(x^{1}\right)\right]$ such that $u_{1}<\hat{u}_{1}$ and $u_{2}>\hat{u}_{2}$. Suppose (contra-hypothesis) that for some $\alpha \in(0,1)$,

$$
\frac{\phi\left(\alpha u_{1}\right)-\phi\left(\alpha \hat{u}_{1}\right)}{\phi\left(\alpha u_{2}\right)-\phi\left(\alpha \hat{u}_{2}\right)} \neq \frac{\phi\left(u_{1}\right)-\phi\left(\hat{u}_{1}\right)}{\phi\left(u_{2}\right)-\phi\left(\hat{u}_{2}\right)}=:-r .
$$

\footnotetext{
16 The proof draws on Moulin's [14, p. 45] proof of Robert's [17] theorem that a social welfare ordering that is additively separable and independent of a common utility scale admits a generalized utilitarian representation with a power function.
} 
Then we have,

$$
\phi^{-1}\left[\frac{1}{1+r} \phi\left(u_{1}\right)+\frac{r}{1+r} \phi\left(u_{2}\right)\right]=\phi^{-1}\left[\frac{1}{1+r} \phi\left(\hat{u}_{1}\right)+\frac{r}{1+r} \phi\left(\hat{u}_{2}\right)\right] .
$$

And (22) implies that

$$
\phi^{-1}\left[\frac{1}{1+r} \phi\left(\alpha u_{1}\right)+\frac{r}{1+r} \phi\left(\alpha u_{2}\right)\right] \neq \phi^{-1}\left[\frac{1}{1+r} \phi\left(\alpha \hat{u}_{1}\right)+\frac{r}{1+r} \phi\left(\alpha \hat{u}_{2}\right)\right] .
$$

But (18) implies (setting $\beta=1 /(1+r)$ )

$$
\begin{aligned}
\phi^{-1}\left[\frac{1}{1+r} \phi\left(\alpha \hat{u}_{1}\right)+\frac{r}{1+r} \phi\left(\alpha \hat{u}_{2}\right)\right]= & \alpha \phi^{-1}\left[\frac{1}{1+r} \phi\left(\hat{u}_{1}\right)+\frac{r}{1+r} \phi\left(\hat{u}_{2}\right)\right], \\
& \text { and } \\
\phi^{-1}\left[\frac{1}{1+r} \phi\left(\alpha u_{1}\right)+\frac{r}{1+r} \phi\left(\alpha u_{2}\right)\right]= & \alpha \phi^{-1}\left[\frac{1}{1+r} \phi\left(u_{1}\right)+\frac{r}{1+r} \phi\left(u_{2}\right)\right],
\end{aligned}
$$

leading to a contradiction. Hence, (21) obtains.

The continuous, increasing solutions of (21) are known (Aczel [1987, Chap. 2]) to be

$$
\phi(u)=C^{+} u^{k^{+}}+D^{+}
$$

for some $C^{+}, k^{+}$in $\mathbb{R}_{++}$and some $D^{+}$in $\mathbb{R} .^{17}$

Case 2. $0 \in\left(\hat{U}_{i_{1}}\left(x^{1}\right), \hat{U}_{i^{1}}\left(x^{1}\right)\right)$. By an analogous argument to the one employed in Case 1 , for $u$ in the sub-interval $\left[0, \hat{U}_{i^{1}}\left(x^{1}\right)\right]$, we obtain $\phi(u)=C^{+} u^{k^{+}}+D^{+}$; and for $u$ in the sub-interval $\left[\hat{U}_{i_{1}}\left(x^{1}\right), 0\right] \subset \mathbb{R}_{-}$, we obtain $\phi(u)=-C^{-}(-u)^{k^{-}}+D^{-}$, for some $C^{-}, k^{-}$in $\mathbb{R}_{++}$and some $D^{-}$ in $\mathbb{R}$. Continuity of $\phi$ implies that $D^{+}=D^{-}=: D$. Thus we obtain:

$$
\phi(u)=\left\{\begin{array}{cc}
C^{+} u^{k^{+}}+D & u \geq 0 \\
-C^{-}(-u)^{k^{-}}+D & u<0
\end{array} .\right.
$$

It remains to show that $k^{+}=k^{-}$and $C^{+}=C^{-}$.

To show that $k^{+}=k^{-}$, we again exploit expression (18). Consider $u^{\prime}, u^{\prime \prime} \in\left(\hat{U}_{i_{1}}\left(x^{1}\right), \hat{U}_{i^{1}}\left(x^{1}\right)\right)$ such that $u^{\prime}<0, u^{\prime \prime}>0$. Then, for any $\alpha, \beta$ in $(0,1)$,

$$
\begin{aligned}
\beta \phi\left(\alpha u^{\prime}\right)+(1-\beta) \phi\left(\alpha u^{\prime \prime}\right)= & -\beta C^{-}\left(-\alpha u^{\prime}\right)^{k^{-}}+(1-\beta) C^{+}\left(\alpha u^{\prime \prime}\right)^{k^{+}}+D \\
& \text { and } \\
\beta \phi\left(u^{\prime}\right)+(1-\beta) \phi\left(u^{\prime \prime}\right)= & -\beta C^{-}\left(-u^{\prime}\right)^{k^{-}}+(1-\beta) C^{+}\left(u^{\prime \prime}\right)^{k^{+}}+D .
\end{aligned}
$$

\footnotetext{
17 Aczel [1987] does not include 0 in the domain of (21). If 0 is excluded, then (21) has a logorithmic solution and a solution in which both $C^{+}$and $k^{+}$are both negative.
} 
Choose $\alpha, \beta$ in $(0,1)$, such that

$$
-\beta C^{-}\left(-\alpha u^{\prime}\right)^{k^{-}}+(1-\beta) C^{+}\left(\alpha u^{\prime \prime}\right)^{k^{+}}>0 \text { and }-\beta C^{-}\left(-u^{\prime}\right)^{k^{-}}+(1-\beta) C^{+}\left(u^{\prime \prime}\right)^{k^{+}}>0 .
$$

Therefore, on the left side of (18) we have

$$
\phi^{-1}\left(\beta \phi\left(\alpha u^{\prime}\right)+(1-\beta) \phi\left(\alpha u^{\prime \prime}\right)\right)=\left(\frac{-\beta C^{-} \alpha^{k^{-}}\left(-u^{\prime}\right)^{k^{-}}+(1-\beta) C^{+}\left(\alpha u^{\prime \prime}\right)^{k^{+}}}{C^{+}}\right)^{1 / k^{+}}
$$

and on the right side of (18) we have

$$
\begin{aligned}
\alpha \phi^{-1}\left(\beta \phi\left(u^{\prime}\right)+(1-\beta) \phi\left(u^{\prime \prime}\right)\right) & =\alpha\left(\frac{-\beta C^{-}\left(-u^{\prime}\right)^{k^{-}}+(1-\beta) C^{+}\left(u^{\prime \prime}\right)^{k^{+}}}{C^{+}}\right)^{1 / k^{+}} \\
& =\left(\frac{-\beta C^{-} \alpha^{k^{+}}\left(-u^{\prime}\right)^{k^{-}}+(1-\beta) C^{+}\left(\alpha u^{\prime \prime}\right)^{k^{+}}}{C^{+}}\right)^{1 / k^{+}}
\end{aligned}
$$

This is possible only if $k^{+}=k^{-}=: k$.

It only remains to show that $C^{+}=C^{-}$. Recall that by redistributive scope and independence over outcome lotteries, there exists an outcome $\hat{x}$ such that $\hat{U}_{i_{1}}(\hat{x})>\hat{U}_{i^{1}}(\hat{x})$. We used this fact to construct $\bar{u}$. Case 2 (i.e., $\left.\bar{u}=0 \in\left(\hat{U}_{i_{1}}\left(x^{1}\right), \hat{U}_{i^{1}}\left(x^{1}\right)\right)\right)$ corresponds to the situation in which $\hat{U}_{i_{1}}(\hat{x})>0>\hat{U}_{i_{1}}\left(x^{1}\right)$.

Recalling the notation we used to define $\bar{u}$, set $\ell_{[\lambda]}:=\lambda[\hat{x}]+(1-\lambda)\left[x^{1}\right]$. By independence over outcome lotteries and our construction, $\hat{U}_{i^{1}}\left(\ell_{[\lambda]}\right)$ is linear and decreasing in $\lambda$, and is positive at $\lambda=0$ and negative at $\lambda=1$; and $\hat{U}_{i_{1}}\left(\ell_{[\lambda]}\right)$ is linear and increasing in $\lambda$, and is negative at $\lambda=0$ and positive at $\lambda=1$. Recall that $\bar{\lambda}$ corresponds to $\bar{u}$; that is, $\ell_{[\bar{\lambda}]}=\bar{\ell}$ and $\hat{U}_{i_{1}}\left(\ell_{[\bar{\lambda}]}\right)=\hat{U}_{i^{1}}\left(\ell_{[\bar{\lambda}]}\right)=0$. By outcome independence, $\bar{\lambda}$ is implicitly given by

$$
\bar{\lambda} \hat{U}_{i_{1}}(\hat{x})+(1-\bar{\lambda}) \hat{U}_{i_{1}}\left(x^{1}\right)=0=\bar{\lambda} \hat{U}_{i^{1}}(\hat{x})+(1-\bar{\lambda}) \hat{U}_{i^{1}}\left(x^{1}\right)
$$

Using $(24)$ we can write for $\lambda>\bar{\lambda}, \hat{U}_{i_{1}}\left(\ell_{[\lambda]}\right)=\hat{U}_{i_{1}}(\hat{x})(\lambda-\bar{\lambda}) /(1-\bar{\lambda})$ and $\hat{U}_{i^{1}}\left(\ell_{[\lambda]}\right)=$ $\hat{U}_{i^{1}}(\hat{x})(\lambda-\bar{\lambda}) /(1-\bar{\lambda})$, and so,

$$
\frac{\hat{U}_{i_{1}}\left(\ell_{[\lambda]}\right)}{\hat{U}_{i^{1}}\left(\ell_{[\lambda]}\right)}=\frac{\hat{U}_{i_{1}}(\hat{x})}{\hat{U}_{i^{1}}(\hat{x})} .
$$

Similarly, for $\lambda<\bar{\lambda}, \hat{U}_{i_{1}}\left(\ell_{[\lambda]}\right)=\hat{U}_{i_{1}}\left(x^{1}\right)(\bar{\lambda}-\lambda) / \bar{\lambda}$ and $\hat{U}_{i^{1}}\left(\ell_{[\lambda]}\right)=\hat{U}_{i^{1}}\left(x^{1}\right)(\bar{\lambda}-\lambda) / \bar{\lambda}$, and so,

$$
\frac{\hat{U}_{i_{1}}\left(\ell_{[\lambda]}\right)}{\hat{U}_{i^{1}}\left(\ell_{[\lambda]}\right)}=\frac{\hat{U}_{i_{1}}\left(x^{1}\right)}{\hat{U}_{i^{1}}\left(x^{1}\right)}
$$


Furthermore, again from equation $(24)$, since $-\hat{U}_{i_{1}}\left(x^{1}\right) / \hat{U}_{i_{1}}(\hat{x})=\bar{\lambda} /(1-\bar{\lambda})=-\hat{U}_{i^{1}}\left(x^{1}\right) / \hat{U}_{i^{1}}(\hat{x})$, we have $\hat{U}_{i_{1}}(\hat{x}) /\left[-\hat{U}_{i^{1}}(\hat{x})\right]=\left[-\hat{U}_{i_{1}}\left(x^{1}\right)\right] / \hat{U}_{i^{1}}\left(x^{1}\right)$. Hence,

$$
\frac{\hat{U}_{i_{1}}\left(\ell_{[\lambda]}\right)}{\hat{U}_{i^{1}}\left(\ell_{[\lambda]}\right)}=\frac{\hat{U}_{i_{1}}\left(x^{1}\right)}{\hat{U}_{i^{1}}\left(x^{1}\right)}
$$

for all $\lambda \neq \bar{\lambda}$.

Also to simplify notation, let $z_{[\gamma]}:=\gamma\left[i_{1}\right]+(1-\gamma)\left[i^{1}\right]$. Using this notation, case 2 implies $V\left(z_{[0]}, \ell_{[0]}\right) \geq V\left(z_{[1]}, \ell_{[1]}\right)>V\left(z_{[1]}, \ell_{[0]}\right)$ and $V\left(z_{[1]}, \ell_{[1]}\right)>V\left(z_{[0]}, \ell_{[1]}\right)$. Let $\bar{v}:=V\left(z_{[1]}, \ell_{[\bar{\lambda}]}\right)=$ $\phi(0)$. By independence over identity lotteries, $V\left(z_{[\gamma]}, \ell_{[\bar{\lambda}]}\right)=\bar{v}$ for all $\gamma$.

By construction, for all $\lambda<\bar{\lambda}, V\left(z_{[0]}, \ell_{[\lambda]}\right)>V\left(z_{[0]}, \ell_{[\bar{\lambda}]}\right)>V\left(z_{[1]}, \ell_{[\lambda]}\right)$; and for all $\lambda>$ $\bar{\lambda}, V\left(z_{[0]}, \ell_{[\lambda]}\right)<V\left(z_{[0]}, \ell_{[\bar{\lambda}]}\right)<V\left(z_{[1]}, \ell_{[\lambda]}\right)$. By the affinity of $V(\cdot, \ell)$ on $\Delta(\mathcal{I})$, for all $\lambda$, $V\left(z_{[\gamma]}, \ell_{[\lambda]}\right)$ is affine in $\gamma$. Thus there exists a unique $\bar{\gamma} \in(0,1)$ such that $V\left(z_{[\bar{\gamma}]}, \ell_{[0]}\right)=\bar{v}$. That is, $\left(z_{[\bar{\gamma}]}, \ell_{[0]}\right) \sim\left(z_{[\bar{\gamma}]}, \ell_{[\bar{\lambda}]}\right)$. An immediate implication of independence over outcome lotteries is that $V\left(z_{[\bar{\gamma}]}, \ell_{[\lambda]}\right)=\bar{v}$ for all $\lambda \leq \bar{\lambda}$. We claim that $V\left(z_{[\bar{\gamma}]}, \ell_{[\lambda]}\right)=\bar{v}$ for all $\lambda$. Suppose not: that is, without loss of generality, there exists a $\lambda>\bar{\lambda}$ such that $V\left(z_{[\bar{\gamma}]}, \ell_{[\lambda]}\right)>\bar{v}$. Then, by independence over outcome lotteries, by mixing with $\left(z_{[\bar{\gamma}]}, \ell_{[0]}\right)$, we would have $V\left(z_{[\bar{\gamma}]}, \ell_{[\lambda]}\right)>\bar{v}$ for all $\lambda>0$, a contradiction. Thus, $V\left(z_{[\bar{\gamma}]}, \ell_{[\lambda]}\right)=\bar{v}$ for all $\lambda$.

We can solve for $\bar{\gamma}$ using the definition of $V$ and the fact that

$$
\bar{\gamma} \phi\left(\hat{U}_{i_{1}}\left(x^{1}\right)\right)+(1-\bar{\gamma}) \phi\left(\hat{U}_{i^{1}}\left(x^{1}\right)\right)=\bar{\gamma} \phi\left(\hat{U}_{i_{1}}(\hat{x})\right)+(1-\bar{\gamma}) \phi\left(\hat{U}_{i^{1}}(\hat{x})\right)
$$

Hence,

$$
\bar{\gamma}=\frac{\phi\left(\hat{U}_{i^{1}}\left(x^{1}\right)\right)-\phi\left(\hat{U}_{i^{1}}(\hat{x})\right)}{\left(\phi\left(\hat{U}_{i_{1}}(\hat{x})\right)-\phi\left(\hat{U}_{i_{1}}\left(x^{1}\right)\right)\right)+\left(\phi\left(\hat{U}_{i^{1}}\left(x^{1}\right)\right)-\phi\left(\hat{U}_{i^{1}}(\hat{x})\right)\right)} .
$$

By the definition of $\bar{\gamma}$, we have

$$
\bar{\gamma} \phi\left[\hat{U}_{i_{1}}\left(\ell_{[\lambda]}\right)\right]+(1-\bar{\gamma}) \phi\left[\hat{U}_{i^{1}}\left(\ell_{[\lambda]}\right)\right]=\bar{v}
$$

for all $\lambda$. By the affinity of $\hat{U}$, we have $\hat{U}_{i_{1}}\left(\ell_{[\lambda]}\right)=\lambda \hat{U}_{i_{1}}(\hat{x})+(1-\lambda) \hat{U}_{i_{1}}\left(x^{1}\right)$ and $\hat{U}_{i^{1}}\left(\ell_{[\lambda]}\right)=$ $\lambda \hat{U}_{i^{1}}(\hat{x})+(1-\lambda) \hat{U}_{i^{1}}\left(x^{1}\right)$. Since this holds for all $\lambda$ and since $\phi$ is differentiable almost everywhere, we have

$$
\bar{\gamma} \phi^{\prime}\left[\hat{U}_{i_{1}}\left(\ell_{[\lambda]}\right)\right]\left(\hat{U}_{i_{1}}(\hat{x})-\hat{U}_{i_{1}}\left(x^{1}\right)\right)+(1-\bar{\gamma}) \phi^{\prime}\left[\hat{U}_{i^{1}}\left(\ell_{[\lambda]}\right)\right]\left(\hat{U}_{i^{1}}(\hat{x})-\hat{U}_{i^{1}}\left(x^{1}\right)\right)=0
$$


at almost all $\lambda$.

Indeed, for all $\lambda \neq \bar{\lambda}$ such that $\hat{U}_{i_{1}}\left(\ell_{[\lambda]}\right)$ and $\hat{U}_{i^{1}}\left(\ell_{[\lambda]}\right)$ lie in $\left(\hat{U}_{i_{1}}\left(x^{1}\right), \hat{U}_{i^{1}}\left(x^{1}\right)\right)$, we can use our homogenous expression for $\phi$ and obtain:

$$
\phi^{\prime}\left[\hat{U}_{i_{1}}\left(\ell_{[\lambda]}\right)\right]=K \phi^{\prime}\left[\hat{U}_{i^{1}}\left(\ell_{[\lambda]}\right)\right]
$$

where $K:=(1-\bar{\gamma})\left(\hat{U}_{i^{1}}\left(x^{1}\right)-\hat{U}_{i^{1}}(\hat{x})\right) /\left[\bar{\gamma}\left(\hat{U}_{i_{1}}(\hat{x})-\hat{U}_{i_{1}}\left(x^{1}\right)\right)\right]$ is a constant (that is, $K$ does not depend on $\lambda$ ).

Since $\phi$ is a power function we have by plugging in (23) to expression (28), for $\lambda>\bar{\lambda}$,

$$
k C^{+}\left(\hat{U}_{i_{1}}\left(\ell_{[\lambda]}\right)\right)^{k-1}=K k C^{-}\left(-\hat{U}_{i^{1}}\left(\ell_{[\lambda]}\right)\right)^{k-1} .
$$

This reduces to

$$
\left[\frac{\hat{U}_{i_{1}}\left(\ell_{[\lambda]}\right)}{-\hat{U}_{i^{1}}\left(\ell_{[\lambda]}\right)}\right]^{k-1}=K \frac{C^{-}}{C^{+}} .
$$

Similarly for for $\lambda<\bar{\lambda}$,

$$
k C^{-}\left(-\hat{U}_{i_{1}}\left(\ell_{[\lambda]}\right)\right)^{k-1}=K k C^{+}\left(\hat{U}_{i^{1}}\left(\ell_{[\lambda]}\right)\right)^{k-1} .
$$

This reduces to

$$
\left[\frac{-\hat{U}_{i_{1}}\left(\ell_{[\lambda]}\right)}{\hat{U}_{i^{1}}\left(\ell_{[\lambda]}\right)}\right]^{k-1}=K \frac{C^{+}}{C^{-}} .
$$

But, by expression (25), the ratio on the left side of both these expressions is equal to $-\hat{U}_{i_{1}}\left(x^{1}\right) / \hat{U}_{i^{1}}\left(x^{1}\right)$ for all $\lambda \neq \bar{\lambda}$. Thus we have shown that $C^{+}=C^{-}$.

Step 5 extends the argument to cover the interval $\left[\hat{U}_{i_{2}}\left(x_{2}\right), \hat{U}_{i^{2}}\left(x_{2}\right)\right]$. So far we have shown that $\phi$ must have the form given in Lemma 9 - that is, an affine transformation of a homogenous function - on the interval $\left[\hat{U}_{i_{1}}\left(x^{1}\right), U_{i^{1}}\left(x^{1}\right)\right]$. We next show that the same function extends over $\left[\hat{U}_{i_{2}}\left(x_{2}\right), \hat{U}_{i^{2}}\left(x_{2}\right)\right]$.

We can repeat Step 2 through Step 4 above focussing on the interval $\left[\hat{U}_{i_{2}}\left(x_{2}\right), \hat{U}_{i^{2}}\left(x_{2}\right)\right]$. The argument is the same except that we need to be careful about the normalization that set $\bar{u}=0$ prior to Step 3. Since we are re-normalizing a second time, we have to keep track of how this second re-normalization is related to the first.

In particular, to be consistent with our notational convention above, let $\left[\hat{U}_{i}\right]_{i \in \mathcal{I}}$ and $\phi$ be the individual levels and $\phi$-function given the normalization that set $\bar{u}=0$ above. In these utility 
units, let the utility level that is analogous to $\bar{u}$ (see expression (8) for the definition) for our analysis of the interval $\left[\hat{U}_{i_{2}}\left(x_{2}\right), \hat{U}_{i^{2}}\left(x_{2}\right)\right]$ be $\bar{u}_{2}$. Denote our re-normalized utility function for each individual $i$ by $\tilde{U}_{i}(\ell):=\hat{U}_{i}(\ell)-\bar{u}_{2}$ (so that the utility level $\bar{u}_{2}$ is re-normalized to zero as before). For each utility level $u$, let $\tilde{u}$ denote the corresponding re-normalized individual utility level and let $\tilde{\phi}$ denote the correspondingly re-normalized $\phi$-function. Then, we can re-normalize $\tilde{\phi}$ such that for all $u$ in $\mathbb{R}, \tilde{\phi}[\tilde{u}]=\tilde{\phi}\left[\left(u-\bar{u}_{2}\right)\right]=\phi[u]$.

By repeating Steps 2 to 4 , we know that $\tilde{\phi}[\tilde{u}]$ must have a form analogous to that in Lemma 9 on the interval $\left[\hat{U}_{i_{2}}\left(x_{2}\right), \hat{U}_{i^{2}}\left(x_{2}\right)\right]$. With slight abuse of notation, we can keep track of the re-normalization by writing

$$
\tilde{\phi}(u)=\left\{\begin{array}{cc}
\tilde{C}\left[u-\bar{u}_{2}\right]^{\tilde{k}}+\tilde{D} & u-\bar{u}_{2} \geq 0 \\
-\tilde{C}\left(-\left(u-\bar{u}_{2}\right)\right)^{\tilde{k}}+\tilde{D} & u-\bar{u}_{2}<0
\end{array} .\right.
$$

By Lemma 6 , we know that $\left[\hat{U}_{i_{2}}\left(x_{2}\right), \hat{U}_{i^{2}}\left(x_{2}\right)\right] \cap\left[\hat{U}_{i_{1}}\left(x^{1}\right), \hat{U}_{i^{1}}\left(x^{1}\right)\right]$ has a non-empty interior. Thus, the $\tilde{\phi}(u)$ and $\phi(u)$ must coincide on this interval. Clearly, if either function were affine, then both functions must be affine and we would be done. Suppose then that $k \neq 1$ and $\tilde{k} \neq 1$. We will show that this implies that $\bar{u}_{2}=0$; that is, the two normalizations must be the same.

Suppose first that the overlap $\left[\hat{U}_{i_{2}}\left(x_{2}\right), \hat{U}_{i^{2}}\left(x_{2}\right)\right] \cap\left[\hat{U}_{i_{1}}\left(x^{1}\right), \hat{U}_{i^{1}}\left(x^{1}\right)\right]$ contains a subinterval in which both $u>0$ and $u-\bar{u}_{2}>0$. Then we know that

$$
\tilde{C}\left[u-\bar{u}_{2}\right]^{\tilde{k}}+\tilde{D}=C u^{k}+D
$$

for all $u$ in that subinterval. Differentiating yields

$$
\tilde{k} \tilde{C}\left[u-\bar{u}_{2}\right]^{\tilde{k}-1}=k C u^{k-1} .
$$

Notice that if $k=\tilde{k}=2$, then we would have $\left[u-\bar{u}_{2}\right] / u=C / \tilde{C}$ and, since the right side is constant, this implies $\bar{u}_{2}=0$. Therefore, assume that $k \neq 2$ or $\tilde{k} \neq 2$. Differentiating again, dividing the second derivative by the first, and rearranging yields

$$
\frac{\left[u-\bar{u}_{2}\right]}{u}=\frac{\tilde{k}-1}{k-1} .
$$

But again the right side is constant, implying that $\bar{u}_{2}=0$. The argument on subintervals where either $u<0$ or $u-\bar{u}_{2}<0$ is similar. 
Since $\bar{u}_{2}=0$ (if $k \neq 1$ ), the first derivative reduces to $u^{k-\tilde{k}}=\tilde{k} \tilde{C} / k C$. But again the right side is a constant. Hence $k=\grave{k}$, so that $\tilde{k} \tilde{C} / k C=u^{k-\tilde{k}}=1$. Therefore $C=\tilde{C}$. Finally, using expression (29), we obtain $D=\tilde{D}$. In other words, the two functions $\phi$ and $\tilde{\phi}$ must be the same.

Step 6 completes the proof by showing that $k=1$. To do this, we invoke our third richness condition, three-player richness.

Recall from the proof of Lemma 9 , for the outcome lottery $\ell_{[\bar{\lambda}]}:=\bar{\lambda}[\hat{x}]+(1-\bar{\lambda})\left[x^{1}\right]$, we had $\hat{U}_{i_{1}}\left(\ell_{[\bar{\lambda}]}\right)=\hat{U}_{i^{1}}\left(\ell_{[\bar{\lambda}]}\right)$. That is, $\left(i^{1}, \ell_{[\bar{\lambda}]}\right) \sim\left(i_{1}, \ell_{[\bar{\lambda}]}\right)$. Hence, by three-player richness, there exists another individual $\hat{\imath}$ such that $\left(\hat{\imath}, \ell_{[\bar{\lambda}]}\right) \nsim\left(i_{1}, \ell_{[\bar{\lambda}]}\right)$. That is, $\hat{U}_{\hat{\imath}}\left(\ell_{[\bar{\lambda}]}\right) \neq 0$. Consider the graphs of $\hat{U}_{i^{1}}\left(\ell_{[\lambda]}\right)$ and $\hat{U}_{\hat{\imath}}\left(\ell_{[\lambda]}\right)$ as functions of $\lambda \in[0,1]$ to $\mathbb{R}$. Both are lines. The first passes through the point $(\bar{\lambda}, 0)$, while the second does not. And, by the definitions of $x^{1}, \hat{x}$ and $i^{1}$, the line $\hat{U}_{i^{1}}\left(\ell_{[\cdot]}\right)$ is strictly decreasing. Suppose that $\hat{U}_{\hat{\imath}}\left(\ell_{[\bar{\lambda}]}\right)>0$ - the argument for the case $\hat{U}_{\hat{\imath}}\left(\ell_{[\bar{\lambda}]}\right)<0$ is similar. Then, we can find $\lambda$ and $\lambda^{\prime}$ such that $0<\lambda<\lambda^{\prime}<\bar{\lambda}$ and such that the vectors $\left(\hat{U}_{i^{1}}\left(\ell_{[\lambda]}\right), \hat{U}_{\hat{\imath}}\left(\ell_{[\lambda]}\right)\right) \gg 0$ and $\left(\hat{U}_{i^{1}}\left(\ell_{\left[\lambda^{\prime}\right]}\right), \hat{U}_{\hat{\imath}}\left(\ell_{\left[\lambda^{\prime}\right]}\right)\right) \gg 0$. Moreover, since $\hat{U}_{\hat{\imath}}\left(\ell_{[\bar{\lambda}]}\right) \neq 0$, these vectors are not colinear.

By the affinity of $\phi^{-1} \circ V$ (Lemma 8), for all $z, \ell, \ell^{\prime}$ and all $\alpha$,

$$
\begin{aligned}
& \phi^{-1}\left[\sum_{i} z_{i} \phi\left[\alpha \hat{U}_{i}(\ell)+(1-\alpha) \hat{U}_{i}\left(\ell^{\prime}\right)\right]\right] \\
= & \alpha \phi^{-1}\left[\sum_{i} z_{i} \phi\left[\hat{U}_{i}(\ell)\right]\right]+(1-\alpha) \phi^{-1}\left[\sum_{i} z_{i} \phi\left[\hat{U}_{i}\left(\ell^{\prime}\right)\right]\right] .
\end{aligned}
$$

In particular, this must hold for $z=(1 / 2)\left[i^{1}\right]+(1 / 2)[\hat{\imath}], \ell_{[\lambda]}$ and $\ell_{\left[\lambda^{\prime}\right]}$. Substituting in these values along with our homogenous functional forms $\phi(u)=C u^{k}+D$ and $\phi^{-1}(v)=[(v-D) / C]^{1 / k}$, the left side of expression (30) becomes:

$$
\begin{aligned}
& \phi^{-1}\left[\frac{1}{2} \phi\left(\alpha \hat{U}_{i^{1}}\left(\ell_{[\lambda]}\right)+(1-\alpha) \hat{U}_{i^{1}}\left(\ell_{\left[\lambda^{\prime}\right]}\right)\right)+\frac{1}{2} \phi\left(\alpha \hat{U}_{\hat{\imath}}\left(\ell_{[\lambda]}\right)+(1-\alpha) \hat{U}_{\hat{\imath}}\left(\ell_{\left[\lambda^{\prime}\right]}\right)\right)\right] \\
= & \phi^{-1}\left[\frac{1}{2} C\left[\left(\alpha \hat{U}_{i^{1}}\left(\ell_{[\lambda]}\right)+(1-\alpha) \hat{U}_{i^{1}}\left(\ell_{\left[\lambda^{\prime}\right]}\right)\right)^{k}+\left(\alpha \hat{U}_{\hat{\imath}}\left(\ell_{[\lambda]}\right)+(1-\alpha) \hat{U}_{\hat{\imath}}\left(\ell_{\left[\lambda^{\prime}\right]}\right)\right)^{k}\right]+D\right] \\
= & \frac{1}{2^{1 / k}}\left[\left(\alpha \hat{U}_{i^{1}}\left(\ell_{[\lambda]}\right)+(1-\alpha) \hat{U}_{i^{1}}\left(\ell_{\left[\lambda^{\prime}\right]}\right)\right)^{k}+\left(\alpha \hat{U}_{\hat{\imath}}\left(\ell_{[\lambda]}\right)+(1-\alpha) \hat{U}_{\hat{\imath}}\left(\ell_{\left[\lambda^{\prime}\right]}\right)\right)^{k}\right]^{1 / k} .
\end{aligned}
$$


And the right side of expression (30) becomes:

$$
\frac{1}{2^{1 / k}}\left(\alpha\left[\left(\hat{U}_{i^{1}}\left(\ell_{[\lambda]}\right)\right)^{k}+\left(\hat{U}_{\hat{\imath}}\left(\ell_{[\lambda]}\right)\right)^{k}\right]^{1 / k}+(1-\alpha)\left[\left(\hat{U}_{i^{1}}\left(\ell_{\left[\lambda^{\prime}\right]}\right)\right)^{k}+\left(\hat{U}_{\hat{\imath}}\left(\ell_{\left[\lambda^{\prime}\right]}\right)\right)^{k}\right]^{1 / k}\right) .
$$

Combining these yields:

$$
\begin{aligned}
& {\left[\left(\alpha \hat{U}_{i^{1}}\left(\ell_{[\lambda]}\right)+(1-\alpha) \hat{U}_{i^{1}}\left(\ell_{\left[\lambda^{\prime}\right]}\right)\right)^{k}+\left(\alpha \hat{U}_{\hat{\imath}}\left(\ell_{[\lambda]}\right)+(1-\alpha) \hat{U}_{\hat{\imath}}\left(\ell_{\left[\lambda^{\prime}\right]}\right)\right)^{k}\right]^{1 / k} } \\
= & \alpha\left[\left(\hat{U}_{i^{1}}\left(\ell_{[\lambda]}\right)\right)^{k}+\left(\hat{U}_{\hat{\imath}}\left(\ell_{[\lambda]}\right)\right)^{k}\right]^{1 / k}+(1-\alpha)\left[\left(\hat{U}_{i^{1}}\left(\ell_{\left[\lambda^{\prime}\right]}\right)\right)^{k}+\left(\hat{U}_{\hat{\imath}}\left(\ell_{\left[\lambda^{\prime}\right]}\right)\right)^{k}\right]^{1 / k} .
\end{aligned}
$$

Notice that if "=" were replaced by " $\leq$ ", then expression (31) becomes the Minkowski inequality. Recall that if the Minkowski inequality holds with equality (and the vectors involved are not colinear), then $k=1$. Since the vectors we chose were not colinear, we have $k=1$, completing the proof.

Remark. Notice that our third richness condition, three-player richness, was only used in the last step (Step 6) of the proof. Specifically, it allowed us to construct vectors that were not colinear, and hence to apply the Minkowski inequality. ${ }^{18}$

The previous step (Step 5) illustrates how our counterexample in section 5 relies on there being two outcomes and two agents. Recall the construction of $\bar{u}$. Starting from the interval $\left[\hat{U}_{i_{1}}\left(x^{1}\right), U_{i^{1}}\left(x^{1}\right)\right]$, redistributive scope ensures there exists an outcome $\hat{x}$ such that $\left(i_{1}, \hat{x}\right) \succ$ $\left(i^{1}, \hat{x}\right)$. Continuity then ensures there exists an outcome lottery $\ell_{[\bar{\lambda}]}$ between $x^{1}$ and $\hat{x}$ such that $\left(i_{1}, \ell_{[\bar{\lambda}]}\right) \sim\left(i^{1}, \ell_{[\bar{\lambda}]}\right)$, and $\bar{u}$ corresponds to the utility level at that lottery. Similarly, starting from the interval $\left[\hat{U}_{i_{2}}\left(x_{2}\right), \hat{U}_{i^{2}}\left(x_{2}\right)\right]$, redistributive scope ensures there exists an outcome $\hat{x}_{2}$ such that $\left(i_{1}, \hat{x}_{2}\right) \succ\left(i^{1}, \hat{x}_{2}\right)$ and continuity ensures there exists an outcome lottery $\ell_{\left[\bar{\lambda}_{2}\right]}$ between $x_{2}$ and $\hat{x}_{2}$ such that $\left(i_{2}, \ell_{\left[\bar{\lambda}_{2}\right]}\right) \sim\left(i^{2}, \ell_{\left[\bar{\lambda}_{2}\right]}\right)$, and $\bar{u}_{2}$ corresponds to the utility level at that lottery. An implication of Step 5 is that if $\bar{u} \neq \bar{u}_{2}$, then $\phi$ is affine. In the example, there are only two outcomes and two agents, hence $\ell_{[\bar{\lambda}]}$ and $\ell_{\left[\bar{\lambda}_{2}\right]}$ must be the same lottery, and therefore $\bar{u}$ and $\bar{u}_{2}$ are trivially equal. But in a world with three agents or three outcomes, such a coincidence is knife edge.

\footnotetext{
18 Even here, we only need this condition if $\hat{U}_{i_{1}}(\hat{x}) \leq 0$. If $\hat{U}_{i_{1}}(\hat{x})>0$, then we have $\left(i_{1}, x^{1}\right) \succ\left(i_{1}, \hat{x}\right)$ and $\left(i^{1}, x^{1}\right) \succ\left(i^{1}, \hat{x}\right)$. In this case, our first richness condition (absence of unanimity) already implies that there exists an $\hat{\imath}$ such that $(\hat{\imath}, \hat{x}) \succ\left(\hat{\imath}, x^{1}\right) \succsim\left(i_{1}, x^{1}\right)$, and hence that $\hat{U}_{\hat{\imath}}\left(\ell_{[\bar{\lambda}]}\right)>0$.
} 


\section{References}

[1] J. Aczél, J, A Short Course on Functional Equations, Dordrecht, D. Reidel, 1987.

[2] A.B. Atkinson, On the Measurement of Inequality, J. Econ. Theory 2 (1970), 244-263.

[3] P.A. Diamond, Cardinal Welfare, Individualistic Ethics, and Interpersonal Comparison of Utility: Comment, J. Polit. Economy 75 (1967), 765-66.

[4] M. Fleurbaey, Assessing Risky Social Situations, J. Polit. Economy 118 (2010), 649-680.

[5] T. Gajdos, F. Kandil, The Ignorant Observer, Soc. Choice Welfare 31 (2008), 193-232.

[6] S. Grant, A. Kajii, B. Polak, Z. Safra, Generalized Utilitarianism and Harsanyi's Impartial Observer Theorem, Econometrica 78 (2010), 1939-71.

[7] S. Grant, A. Kajii, B. Polak, Z. Safra, A Generalization of Harsanyi's Impartial Observer Theorem," mimeo (2010).

[8] A. Harel, Z. Safra, U. Segal, Ex-Post Egalitarianism and Legal Justice, The J. Law Econ. Organ. 21 (2005), 57-75.

[9] G.H. Hardy, J.E. Littlewood, G. Pólya, Inequalities, Cambridge, Cambridge University Press, 1934.

[10] J.C. Harsanyi, Cardinal Utility in Welfare Economics and in the Theory of Risk-Taking. J. Polit. Economy 61 (1953), 434-5.

[11] J.C. Harsanyi, Cardinal Welfare, Individualistic Ethics, and Interpersonal Comparison of Utility: J. Polit. Economy 63 (1955), 309-21.

[12] E. Karni, J.A. Weymark, An Informationally Parsimonious Impartial Observer Theorem, Soc. Choice Welfare 15 (1998), 321-332.

[13] A.A. Milne, The House at Pooh Corner, New York, E.P. Dutton, 1928.

[14] H. Moulin, Axioms of Cooperative Decision Making, Cambridge: Cambridge University Press, 1988.

[15] J.E. Roemer, Utilitarianism, The Difference Principle, and the Veil of Ignorance: An Application of the Theory of Social Situations, in: R. Selten (ed.) Rational Interaction: Essays in Honor of John C. Harsanyi, Springer-Verlag, 1992, 337-51.

[16] M. Rothschild, J.E. Stilitz, Increasing Risk I: A Definition, J. Econ. Theory 2 (1970), $225-243$.

[17] K. Roberts, Possibility Theorems with Interpersonally Comparable Welfare Levels, Rev. Econ. Stud., 47 (1980), 409-420.

[18] J.A. Weymark, A reconsideration of the Harsanyi-Sen debate on utilitarianism, in: J. Elster, J.E. Roemer (eds) Interpersonal comparisons of well-being. Cambridge University Press, Cambridge, 1991, pp 255-320. 